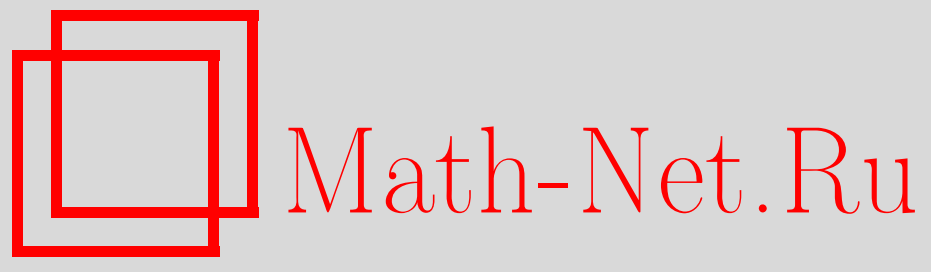

Ч.-Ю. Юнг, Р. Темам, Теория пограничного слоя для уравнений конвекции-диффузии в круге, УМН, 2014, том 69 , выпуск $3,43-86$

DOI: https://doi.org/10.4213/rm9584

Использование Общероссийского математического портала Math-Net.Ru подразумевает, что вы прочитали и согласны с пользовательским соглашением http://www . mathnet.ru/rus/agreement

Параметры загрузки:

IP: 35.174 .16 .151

26 апреля 2023 г., 14:44:44

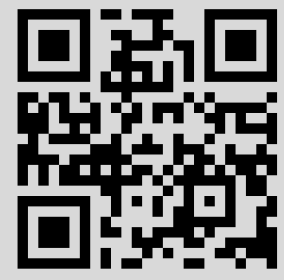




\title{
Теория пограничного слоя для уравнений конвекции-диффузии в круге
}

\author{
Ч.-Ю. Юнг, Р. Темам
}

Статья посвящена теории пограничного слоя для сингулярно возмущенных уравнений конвекции-диффузии в единичном круге. В контексте рассматриваемых здесь уравнений появляются две характеристические точки, $( \pm 1,0)$, и в этих точках могут возникнуть сингулярности в зависимости от поведения в них заданной функции $f$, а именно, от уплощения или совместности $f$ в этих точках в смысле, объясняемом ниже. Две наши предыдущие статьи были посвящены двум частным случаям: в [24] рассматривался случай, когда функция $f$ является достаточно плоской в характеристических точках, так называемый совместный случай; в [25] рассматривался типичный несовместный случай $(f-$ полином $)$. В данной обзорной статье воспроизводятся существенные результаты из [24], [25], а также рассматривается общий случай $(f-$ не плоская и не полиномиальная), для которого дополнительно вводятся новые специальные функции пограничного слоя, имеющие параболический тип.

Библиография: 49 названий.

Ключевые слова: пограничные слои, сингулярные возмущения, задачи с преобладанием конвекции, характеристические точки, параболические пограничные слои.

DOI: $10.4213 / \mathrm{rm} 9584$

\section{СОДЕРЖАНИЕ}

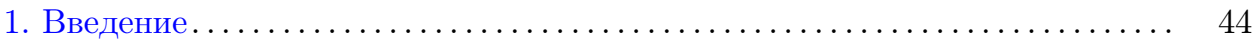

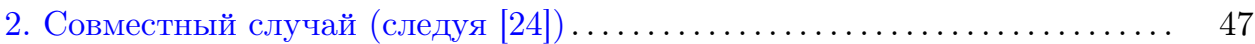

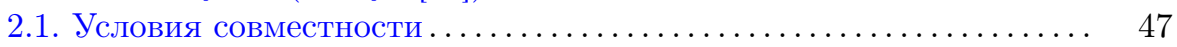

2.2. Граничные выпрямляющие координаты ............... 50

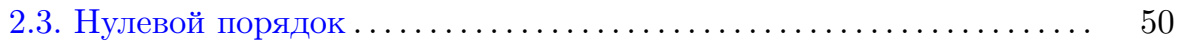

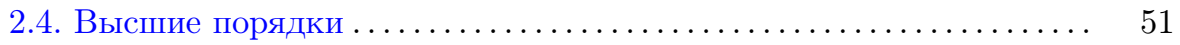

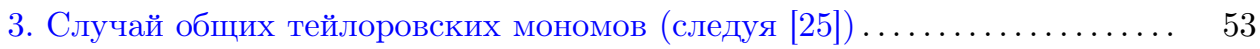

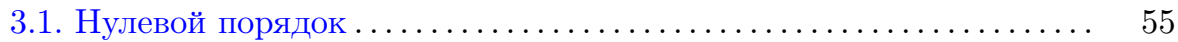

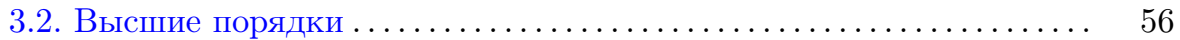

4. Параболические пограничные слои в характеристических точках ..... 57

Работа выполнена при поддержке NSF (грант DMS 1206438), а также Фонда исследований Университета Индианы и гранта NRF-2012R1A1B3001167 Национального фонда исследований (NRF) Кореи, финансируемого Правительством Кореи (MSIP).

(C) Ч.-Ю. Юнг, Р. Темам, 2014 


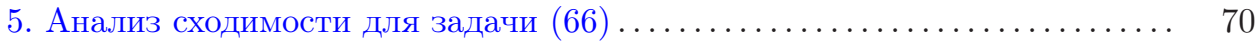

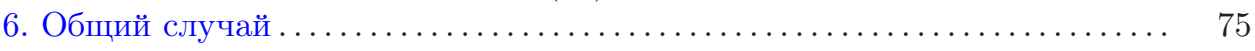

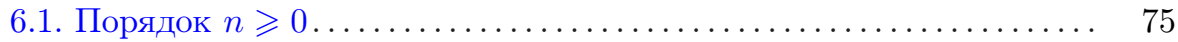

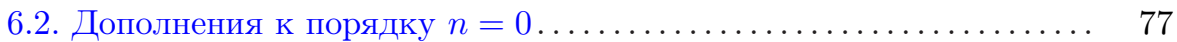

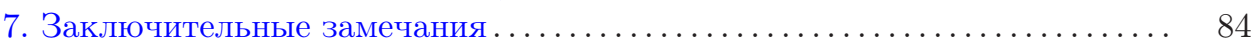

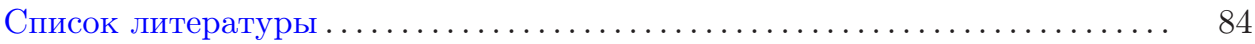

\section{1. Введение}

Вопрос о поведении жидкости, когда вязкость мала, остается важной проблемой в науке и инженерных исследованиях при изучении турбулентных пограничных слоев. Некоторые продвижения были сделаны недавно в случае, когда граница жидкости не является характеристической [43], [16]. Когда граница оказывается характеристической, задача поведения решений уравнений Навье-Стокса для несжимаемой жидкости с малой вязкостью остается одной из главных проблем нелинейного анализа. Даже в случае двух измерений, когда известно, что как уравнения Навье-Стокса для несжимаемой жидкости, так и уравнения Эйлера обладают единственным гладким решением для всех времен (см. [26] и, например, [41]), вопрос сходимости решений уравнений Навье-Стокса к соответствующим решениям уравнений Эйлера при вязкости, стремящейся к 0, остается нерешенным и часто поднимается некоторыми авторами. Что касается прикладных наук, то нужды аэронавтики и астронавтики привели к возникновению ряда эмпирических законов, начиная с работ Прандтля и фон Кармана [35], [36], [47], продолженных многими другими исследователями. Тем не менее очевидно, что существенный прогресс может быть достигнут на пути лучшего понимания свойств пограничных слоев на аэродинамических поверхностях и возможного их устранения при помощи теории контроля, что является предметом настоящего исследования.

Упрощенная модель, которая будет рассмотрена в настоящей статье, соответствует двумерной стационарной задаче, линеаризованной около скорости $(0,-1)$; уравнения, используемые для каждой компоненты скорости или для температуры, составляют термо-гидравлическую задачу. Заметим, что даже для упрощенной формы, рассмотренной в данной статье, в теоретической части анализа пограничных слоев, возникающих в этой задаче, лишь небольшой прогресс был достигнут со времени ранних работ Левинсона [28], Экхауза и де Ягера [11], Грасмана [13]; о более недавних результатах см. работы Верхалста [45] и Юнга и Темама [24], [25]. Эти теоретические работы будут рассмотрены ниже. С вычислительной стороны эта упрощенная задача остается достаточно серьезной; см., например, обзорную статью [40]. В сущности, мы надеемся, что, когда будет достигнуто лучшее понимание свойств пограничных слоев, возникающих в данной задаче, появится возможность усовершенствования численного решения путем комбинирования численных методов с анализом пограничного слоя, как это делалось в более простых случаях, например в [18], [20], [21]. Заметим также, что, как мы предполагаем, при лучшем понимании этого явления будут введены параболические пограничные слои, которые послужат основой для изучения явлений типа так называемых "шепчущих стенок" (см., например, [1]). И, наконец, в качестве дополнительной мотивации данной работы упомянем, 
что, заменяя $-u_{y}^{\varepsilon}$ на $-u^{\varepsilon}$ в уравнении (1) ниже, мы получим уравнение типа Гельмгольца $\left(\Delta+k^{2}\right) u^{k}=\tilde{f}, k \gg 1$, описывающее задачу, которая является центральной в классической акустике и оптике, а также в современной теории лазеров и нелинейной оптике [1], [5], [27]. Это дополнительное наблюдение несомненно потребует дальнейшего развития.

Возвращаясь к (1), мы надеемся в итоге, что сложность задачи, изучаемой ниже, свидетельствует о сложности соответствующего явления, которое может возникнуть в механике жидкости (так же как, возможно, в акустике, магнитогидродинамике и оптике).

В данной статье будут рассмотрены следующие сингулярно возмущенные задачи в круговой области:

$$
\begin{cases}L_{\varepsilon} u^{\varepsilon}:=-\varepsilon \Delta u^{\varepsilon}-u_{y}^{\varepsilon}=f & \text { в } D, \\ u^{\varepsilon}=0 & \text { на } \partial D,\end{cases}
$$

где $0<\varepsilon \ll 1, D$ - единичный диск с центром $(0,0)$ и рассматриваются произвольные гладкие функции $f=f(x, y)$ без каких-либо ограничений поведения в характеристических точках $( \pm 1,0)$.

Нижний и верхний полукруги обозначаются через $C_{u}(x)=\sqrt{1-x^{2}}$ и $C_{l}(x)=$ $-\sqrt{1-x^{2}}$ соответственно.

Как отмечено в более ранних статьях [24], [25], предполагается, что предельная функция $u^{0}$ является решением краевой задачи

$$
\begin{cases}-u_{y}^{0}=f & \text { в } D \\ u^{0}=0 & \text { на } \Gamma_{u}\end{cases}
$$

где граница втекания обозначается $\Gamma_{u}=\left\{(x, y) \mid x^{2}+y^{2}=1, y>0\right\}$. Решение может быть найдено в явной форме и имеет вид

$$
u^{0}(x, y)=\int_{y}^{C_{u}(x)} f(x, s) d s, \quad(x, y) \in D .
$$

Выбор граничного условия втекания $(2)_{2}\left(u^{0}=0\right.$ на $\Gamma_{u}$, а не, скажем, $u^{0}=0$ на $\left.\Gamma_{l}=\left\{(x, y) \mid x^{2}+y^{2}=1, y<0\right\}\right)$ оправдывается направлением характеристик на границе и подтверждается теоремами сходимости, приведенными ниже (см., например, теорему 4).

Тем не менее в общем случае предел может быть сингулярным; действительно, $(2)_{2}$ влечет за собой по непрерывности, что тангенциальная производная $u_{y}^{0}$ обращается в нуль в $( \pm 1,0)$, что несовместимо с $(2)_{1}$, если $f( \pm 1,0)$ не обращается в нуль. В [24] подробно обсуждается степень регулярности $u^{0}$, зависящая от уплощения $f$ в точках $( \pm 1,0)$. Более общо, вводится полное внешнее разложение $u^{\varepsilon} \sim \sum_{j=0}^{\infty} \varepsilon^{j} u^{j}$ и обсуждается регулярность $u^{j}$ в зависимости от уплощения $f$. Главные результаты воспроизводятся в разделе 2, который также воспроизводит (взятое из [24]) полное асимптотическое разложение $u^{\varepsilon}$ для данного случая.

Сходимость $u^{\varepsilon}$ к $u^{0}$ в $L^{2}(D)$ при $\varepsilon \rightarrow 0$ изучена в предыдущих статьях. Среди прочих, читатель может обратиться к работам К. Бардоса [2], Н. Левинсона [28], В. Экхауза, Е. М. де Ягера [11] и Н. М. Темме [44]. Тем не менее, 
в отличие от настоящей статьи, эти результаты неверны в окрестности характеристических точек $( \pm 1,0)$. Более того, в [28] и [11] используется принцип максимума, без которого нам здесь удается обойтись; это позволяет верить, что наши методы и результаты применимы к случаям, когда принцип максимума не выполняется, как, например, для систем и уравнений более высоких порядков. Начиная настоящую работу и более раннюю работу [24], мы не знали о существовании обширной литературы по этой проблеме - это было нам указано одним из рецензентов статьи [25]; см. [13], [45] и ссылки там. Настоящая работа не отменяется этими результатами, и ниже будет объяснено, как она соотносится с [13] и в каком смысле имеет преимущества по сравнению с этой работой.

Конечно, имеется обширная литература, посвященная проблемам сингулярных возмущений уравнений в частных производных, абстрактных эллиптических или параболических уравнений или уравнений, относящихся к механике жидкости; см., например, [6], [7], [3], [8], [9], [10], [12], [17], [14], [15], [19], [29], [32], [34], [40], [39], [43], [46], [1], [4], [5], [27], [30], [31], [33], [37], [38], [42], [20], [22], [23], [45], [48], [49] и ссылки в этих работах.

Возвращаясь к (1), общий случай для (1) изучается разложением $f$ в три шага в силу линейности:

- Рассматривая сначала разложение Тейлора $\widehat{f}$ функции $f$ (до некоторого порядка), можно рассмотреть $f-\widehat{f}$ как достаточно плоскую функцию в $( \pm 1,0)$. Этот случай, как уже говорилось, изучен в [24].

- В [25] рассмотрен случай, когда $f$ заменена типичным мономом тейлоровского разложения $\widehat{f}$ функции $f$. В общем случае $\widehat{f}$ не является плоской в $( \pm 1,0)$, т. е. несовместна, и ожидается, что имеют место некоторые особенности предельной функции $u^{0}$, как указано в [24]. По этой причине, сначала рассмотрим $\rho(x) \check{\rho}(x) \widehat{f}$, где $\rho(x), \check{\rho}(x)$ являются гладкими обрезанными функциями, обращающимися в нуль в малой окрестности соответственно точек $x=1,-1$ и $y=0$ (см. (43)-(45) ниже), и особенности $u^{j}$ можно выделить. Подробности даны в разделе 3 с воспроизведением главных результатов [25]. Затем в [25] рассматривались $(1-\rho(x) \check{\rho}(x)) \widehat{f}$ с малым носителем размера $\sigma$ около точек $( \pm 1,0)$, и, сравнивая $\sigma$ и $\varepsilon$, мы доказываем, что соответствующее решение $u^{\varepsilon}$ мало.

- В данной статье показано, как обращаться с $f$ общего вида. Запишем $f=f-\widehat{f}+\rho(x) \check{\rho}(x) \widehat{f}+(1-\rho(x) \check{\rho}(x)) \widehat{f}$. Из [24] и [25] следует, что изучение $f$ сводится к изучению $f-\widehat{f}$ или $\rho(x) \check{\rho}(x) \widehat{f}$. Сконцентрируемся на случае, когда $f$ заменяется на $(1-\rho(x) \check{\rho}(x)) \hat{f}$, что создает так называемые пограничные слои, которые строятся в данной статье. Отсюда будет следовать поведение $f$ в общем случае. Заметим, что можно было бы рассмотреть разложение $f=\rho(x) \check{\rho}(x) f+(1-\rho(x) \check{\rho}(x)) f$. Но, так как $\widehat{f}$ - моном тейлоровского разложения, т. е. $(1-x)^{p} y^{q}$, относительно легко проанализировать соответствующие решения и, в зависимости от порядка монома $\widehat{f}$, возможно также систематически и полностью изучать их поведение, что невозможно сделать с общей функцией $(1-\rho \check{\rho}) f$. Более того, рассмотрение мономов позволяет использовать геометрию нашей области, т. е. круга $C_{u}(x)$; так, например, $2^{-p} C_{u}(x)^{2 p} y^{q} \approx(1-x)^{p} y^{q}$ 
вблизи $(x, y)=(1,0)$ и особенности, производимые $C_{u}(x)=\sqrt{1-x^{2}}$, могут быть лучше поняты.

В заключение мы хотели бы выделить одно из главных достижений этой статьи. Для того чтобы построить асимптотические разложения решений при стремящейся к нулю вязкости и вывести априорно пригодные оценки "ошибок", необходимы конкретные определения функций пограничного слоя, решений аналога уравнений Прандтля для данной задачи. Такие оценки могут быть легко выведены, когда решения уравнений Прандтля (уравнений пограничного слоя) известны в явной форме, это решения "простой" эллиптической или параболической задачи типа уравнения притока тепла; см., например, [21], [20], [39], [1] и [34]; см. также [13], где решение уравнений пограничного слоя выражается через функции Эйри. В настоящей работе уравнения пограничного слоя, которые возникают при изучении уравнения (1), когда $f$ заменяется на $(1-\rho \check{\rho}) \widehat{f}$, как указано выше, не могут быть решены в явной форме. Тем не менее и это является одним из достижений данной статьи, удается вывести различные оценки функций пограничного слоя, используя подходящие адаптированные ядра, обозначенные в тексте $M_{l, d}$ : см. (95) и леммы 8 и 9 . Мы надеемся, что эта новая техника может оказаться полезной в других случаях, и намереваемся использовать ее в дальнейших работах.

Эта статья посвящена памяти Марка Вишика, в знак высокой оценки его фундаментального вклада во многие области анализа, особенно в теорию сингулярных возмущений (в [46] и других работах), и как свидетельство теплых воспоминаний одного из авторов (Р.T.) о многочисленных визитах и встречах с Вишиком в Москве, Париже, Берлине и Блумингтоне.

\section{2. Совместный случай (следуя [24])}

Прежде чем продолжить, представим здесь полное внешнее асимптотическое разложение $u^{\varepsilon}: u^{\varepsilon} \sim \sum_{j=0}^{\infty} \varepsilon^{j} u^{j}$. Подставляя это разложение в $(1)_{1}$, формально получим для $j=0,1, \ldots$

$$
\begin{cases}-u_{y}^{j}=\Delta u^{j-1} & \text { в } D \\ u^{j}=0 & \text { на } \Gamma_{u}\end{cases}
$$

Здесь для удобства было записано $\Delta u^{-1}=f$. Решения $u^{j}$ задачи (4), обращающиеся в нуль на $\Gamma_{u}$, легко найти:

$$
u^{j}(x, y)=\int_{y}^{C_{u}(x)} \Delta u^{j-1}(x, s) d s, \quad(x, y) \in D,
$$

где $C_{u}(x)=\sqrt{1-x^{2}}$.

2.1. Условия совместности. Для того чтобы вывести свойства регулярности $u^{j}$, потребуется следующий тип условий совместности:

$$
\frac{\partial^{p+q} f}{\partial x^{p} \partial y^{q}}=0 \quad \text { в точках }( \pm 1,0), \quad 0 \leqslant 2 p+q \leqslant \mu+3 j, \quad p, q, j \geqslant 0 ;
$$

$\mu$ будет определено ниже. 
Свойства регулярности $u^{j}$, вытекающие из таких условий совместности, будут ясны после результатов следующих двух технических лемм, доказанных в [24].

Лемма 1. Предположим, что

$$
\frac{\partial^{\alpha+\beta} g(x, y)}{\partial x^{\alpha} \partial y^{\beta}}=0 \quad \text { в }( \pm 1,0), \quad 0 \leqslant 2 \alpha+\beta \leqslant \gamma-1, \quad \gamma \geqslant 1, \quad \alpha, \beta \geqslant 0,
$$

где $g(x, y)$ принадлежст $\mathscr{C}^{\gamma}(\bar{D})$, a $D$, определенное после $(1)$, является единичным диском с иентром в начале координат. Тогда функиия

$$
\frac{g\left(x, C_{u}(x)\right)}{C_{u}^{\gamma}(x)}
$$

ограничена для всех $x \in(-1,1)$.

Целью следующей леммы является вывод подходящей формы $u^{j}$, их производных и их первообразных. Затем, используя некоторые из условий совместности (6), мы выведем свойства регулярности $u^{j}$ (см. лемму 3 ниже). Сначала введем обозначение

$$
\frac{\partial^{-1} g}{\partial y^{-1}}(x, y)=\int_{y}^{C_{u}(x)} g(x, s) d s
$$

так что, например,

$$
u^{0}=\frac{\partial^{-1} f}{\partial y^{-1}}
$$

Лемма 2. Для всех $i, j \geqslant 0$ u $m \in \mathbb{Z}$ функиии $u^{j}$, определенные в (5), и их производные выражаются следующим образом:

$$
\begin{aligned}
\left\{\frac{\partial^{m}}{\partial y^{m}}\left[\frac{\partial^{i} u^{j}}{\partial x^{i}}\right]\right\}(x, y)= & \sum_{l+s \leqslant i+2 j-1, l, s \geqslant 0} g_{l i m s}^{j}(x, y) \frac{\partial^{l+s} f}{\partial x^{l} \partial y^{s}}\left(x, C_{u}(x)\right) \\
& +\sum_{k=0}^{j} c_{k i m}^{j}\left\{\frac{\partial^{m-j+2 k-1}}{\partial y^{m-j+2 k-1}}\left[\frac{\partial^{i+2 j-2 k} f}{\partial x^{i+2 j-2 k}}\right]\right\}(x, y),
\end{aligned}
$$

əəe

$$
\left|\frac{\partial^{r+q}}{\partial x^{r} \partial y^{q}} g_{\text {lims }}^{j}(x, y)\right| \leqslant \kappa C_{u}(x)^{-(-1+3 j+2 r+2 i-2 l-s)} \quad \forall r, q \geqslant 0 .
$$

$K$ тому же, если $m \geqslant 0$, mо $g_{\text {lims }}^{j}(x, y)=g_{\text {lims }}^{j}(x)$ и коэфбициенты $c_{k i m}^{j} u$ $\kappa$ являются константами, причем $\kappa$ зависит от $r, j, l, i, m, s, q$.

Следующие свойства регулярности $u^{j}$ при условиях совместности (6) не сформулированы явно в [24], но они легко следуют из приведенных там рассуждений. 
ЛЕмма 3. Предположим, что выполняются условия совместности (6). Для всех $i, j, m \geqslant 0$ функиии и $u^{j}$ и их производные оцениваются следующим образом: для $w \in H_{0}^{1}(D)$

$$
\begin{aligned}
& \left|\int_{D} \frac{\partial^{i+m} u^{j}}{\partial x^{i} \partial y^{m}} w d x d y\right| \\
& \leqslant \kappa \begin{cases}|w|_{L^{2}(D)}, & \text { если выполнено }(6) c \mu=-2+2 i, \\
|w|_{H^{1}(D)}^{1 / 2}|w|_{L^{2}(D)}^{1 / 2}, & \text { если выполнено }(6) c \mu=-3+2 i, \\
|w|_{H^{1}(D)}, & \text { если выполнено }(6) \text { с } \mu=-4+2 i .\end{cases}
\end{aligned}
$$

Если выполнено (6) с $\mu=-2+2 i$, имеем следуюшую поточечную оценку:

$$
\left|\frac{\partial^{i+m} u^{j}}{\partial x^{i} \partial y^{m}}\right| \leqslant \kappa
$$

ДокАЗАТЕльство. Заметим, что для $w \in H_{0}^{1}(D)$

$$
\begin{gathered}
\left|\int_{D} \frac{\partial^{i+m} u^{j}}{\partial x^{i} \partial y^{m}} w d x d y\right| \leqslant \kappa|w|_{L^{2}(D)}+\kappa \sum_{l+s \leqslant i+2 j-1, l, s \geqslant 0} \mid \int_{D} \frac{\partial^{l+s} f}{\partial x^{l} \partial y^{s}}\left(x, C_{u}(x)\right) \\
\times C_{u}(x)^{-(-k-1+3 j+2 i-2 l-s)} \cdot C_{u}(x)^{-k} w d x d y \mid .
\end{gathered}
$$

Сначала оценим $\left|C_{u}(x)^{-k} w\right|_{L^{1}(D)}$ :

$$
\left|C_{u}(x)^{-k} w\right|_{L^{1}(D)} \leqslant \kappa \begin{cases}|w|_{L^{2}(D)}, & \text { если } k=0, \\ \left|w_{x}\right|_{L^{2}(D)}^{1 / 2}|w|_{L^{2}(D)}^{1 / 2}, & \text { если } k=1, \\ \left|w_{x}\right|_{L^{2}(D)}, & \text { если } k=2 .\end{cases}
$$

Действительно, для $k=0$ это очевидно. Для $k=2$ это следует из неравенства Харди. В случае $k=1$, снова благодаря неравенству Харди, для $w \in H_{0}^{1}(D)$ находим, что

$$
\begin{aligned}
& \left|\int_{D} \frac{w}{C_{u}(x)} d x d y\right| \leqslant \kappa\left(\int_{D} \frac{w^{2}}{1-x^{2}} d x d y\right)^{1 / 2} \\
& \quad \leqslant \kappa\left|\frac{w}{1-x}+\frac{w}{1+x}\right|_{L^{2}(D)}^{1 / 2}|w|_{L^{2}(D)}^{1 / 2} \leqslant \kappa\left|w_{x}\right|_{L^{2}(D)}^{1 / 2}|w|_{L^{2}(D)}^{1 / 2} .
\end{aligned}
$$

Ограничим некоторой положительной постоянной функцию $\frac{\partial^{l+s} f}{\partial x^{l} \partial y^{s}}\left(x, C_{u}(x)\right) \times$ $C_{u}(x)^{-(-k-1+3 j+2 i-2 l-s)}$. Для этого, вследствие леммы 1 , потребуем, чтобы $\frac{\partial^{p+q}}{\partial x^{p} \partial y^{q}}\left(\frac{\partial^{l+s} f}{\partial x^{l} \partial y^{s}}\right)=0$ в $( \pm 1,0), 0 \leqslant 2 p+q \leqslant-k-2+3 j+2 i-2 l-s$, что гарантируется следующими условиями совместности:

$$
\frac{\partial^{p+q} f}{\partial x^{p} \partial y^{q}}=0 \quad \text { в }( \pm 1,0), \quad 0 \leqslant 2 p+q \leqslant-k-2+3 j+2 i, \quad p, q \geqslant 0 .
$$

Заметим, что если выполнены условия совместности (6), то (16) выполняется при $\mu=-k-2+2 i$. Таким образом, утверждения леммы следуют из (13) и (14). В частности, если выполняется (6) с $\mu=-2+2 i$, то (10b) ограничено некоторой положительной постоянной, что означает выполнение поточечной оценки (12). Лемма доказана. 
2.2. Граничные выпрямляющие координаты. Для того чтобы разрешить расхождение граничных данных и определить корректоры, удобнее рассматривать данную задачу в граничных выпрямляющих координатах. Пусть $\xi$ - расстояние до границы $\partial D$, считаемое в направлении внутренней нормали, и $\eta$ - длина дуги $\partial D$ с началом в точке $(x, y)=(1,0)$, считаемая в направлении против часовой стрелки.

При использовании граничных выпрямляющих координат $x=(1-\xi) \cos \eta$, $y=(1-\xi) \sin \eta, \xi=1-r$, где $r-$ расстояние до центра $(0,0)$ и $\eta-$ полярный угол с осью $O x$, т. е. $\xi=1-\sqrt{x^{2}+y^{2}}$ и $\eta=\operatorname{arctg}(y / x)$, область $D$ отображается на область

$$
D^{*}=\{(\eta, \xi) \in(0,2 \pi) \times(0,1)\},
$$

а дифференциальные операторы имеют вид

$$
\begin{aligned}
\frac{\partial}{\partial x} & =-\cos \eta \frac{\partial}{\partial \xi}-\frac{\sin \eta}{1-\xi} \frac{\partial}{\partial \eta} \\
\frac{\partial}{\partial y} & =-\sin \eta \frac{\partial}{\partial \xi}+\frac{\cos \eta}{1-\xi} \frac{\partial}{\partial \eta} .
\end{aligned}
$$

Преобразуем также дифференциальные операторы в (1) в

$$
\begin{aligned}
L_{\varepsilon} u^{\varepsilon} & =-\varepsilon \Delta u^{\varepsilon}-u_{y}^{\varepsilon} \\
& =-\frac{\varepsilon}{(1-\xi)^{2}} \frac{\partial^{2} u^{\varepsilon}}{\partial \eta^{2}}+\frac{\varepsilon}{1-\xi} \frac{\partial u^{\varepsilon}}{\partial \xi}-\varepsilon \frac{\partial^{2} u^{\varepsilon}}{\partial \xi^{2}}+\sin \eta \frac{\partial u^{\varepsilon}}{\partial \xi}-\frac{\cos \eta}{1-\xi} \frac{\partial u^{\varepsilon}}{\partial \eta} .
\end{aligned}
$$

2.3. Нулевой порядок. Построим корректор $\theta^{0}$, который корректирует граничные значения в $\xi=0, \pi<\eta<2 \pi$. Рассматривая растягивающую переменную $\bar{\xi}=\xi / \varepsilon$, мы можем определить доминирующие дифференциальные операторы и приходим к следующему уравнению для первого корректора $\theta^{0}$ :

$$
\begin{cases}-\frac{\partial^{2} \theta^{0}}{\partial \bar{\xi}^{2}}+\sin \eta \frac{\partial \theta^{0}}{\partial \bar{\xi}}=0 & \text { для } 0<\bar{\xi}<\infty, \pi<\eta<2 \pi, \\ \theta^{0}=-u^{0}(\cos \eta, \sin \eta) & \text { в } \bar{\xi}=0, \\ \theta^{0} \rightarrow 0 & \text { при } \bar{\xi} \rightarrow \infty .\end{cases}
$$

Отсюда удается получить решение в явной форме:

$$
\theta^{0}=-u^{0}(\cos \eta, \sin \eta) \exp \left(\frac{\sin \eta}{\varepsilon} \xi\right) \chi_{[\pi, 2 \pi]}(\eta),
$$

где $\chi_{A}$ - характеристическая функция для $A$. Используя обрезывающую функцию, запишем $\theta^{0}$ в приближенном виде:

$$
\bar{\theta}^{0}(\eta, \xi)=\theta^{0}(\eta, \xi) \delta(\xi)
$$

где $\delta=\delta(\xi)$ - гладкая обрезывающая функция такая, что $\delta(\xi)=1$ при $\xi \in$ $[0,1 / 4]$ и $\delta(\xi)=0$ при $\xi \in[1 / 2,1]$.

Поскольку $\theta^{0}$, подобно $u^{0}$, обращается в нуль при $\eta=\pi, 2 \pi$, то $\theta^{0}$ непрерывна и является кусочно гладкой в $\bar{D}$, откуда заключаем, что $\theta^{0}, \bar{\theta}^{0} \in H^{1}(D)$. Заметим, что из (3) следует

$$
u^{0}(\cos \eta, \sin \eta)=\int_{\sin \eta}^{-\sin \eta} f(\cos \eta, s) d s, \quad \pi<\eta<2 \pi,
$$

и $u^{0}+\bar{\theta}^{0} \in H_{0}^{1}(D)$. 
Тогда имеем следующие результаты о сходимости, доказанные в [24]. Используется весовая энергетическая норма

$$
\|\cdot\|_{\varepsilon}=\sqrt{\varepsilon}|\cdot|_{H^{1}(D)}+|\cdot|_{L^{2}(D)} .
$$

Теорема 1. Пусть $u^{\varepsilon}$ и $u^{0}$ - решения задач (1) $и$ (2) соответственно, $u$ пусть $\bar{\theta}^{0}$ - приближенная форма $\theta^{0}$, определенная в (22). Если выполняются условия совместности (6) с $\mu=0, j=0$, m.е.

$$
f=0 \quad \text { в }( \pm 1,0),
$$

то справедливы следующие оченки:

$$
\left\|u^{\varepsilon}-u^{0}-\bar{\theta}^{0}\right\|_{\varepsilon} \leqslant \kappa \varepsilon^{1 / 2}
$$

u, таким образом,

$$
\left|u^{\varepsilon}-u^{0}\right|_{L^{2}(D)} \leqslant \kappa \varepsilon^{1 / 2}
$$

где $\bar{\theta}^{0}$ - корректор из (22).

Теорема 2. Пусть $u^{\varepsilon} u u^{0}$ - решения задач (1) $и$ (2) соответственно, $и$ пусть $\bar{\theta}^{0}$ - приближенная форма $\theta^{0}$, определенная в (22). Если выполняются условия совместности (6) с $\mu=1, j=0$, m.е.

$$
f=\frac{\partial f}{\partial y}=0 \quad \text { в }( \pm 1,0)
$$

то справедливы следующие оценки:

$$
\left\|u^{\varepsilon}-u^{0}-\bar{\theta}^{0}\right\|_{\varepsilon} \leqslant \kappa \varepsilon^{3 / 4}
$$

где $\bar{\theta}^{0}-$ корректор из (22).

2.4. Высшие порядки. По аналогии с (20), введем корректор пограничного слоя $u^{\varepsilon} \sim \sum_{j=0}^{\infty} \varepsilon^{j} \theta^{j}$. Используя разложения $(1-\xi)^{-1}=\sum_{k=0}^{\infty} \xi^{k},(1-\xi)^{-2}=$ $\sum_{k=0}^{\infty}(k+1) \xi^{k}$, где $\xi=\bar{\xi} \varepsilon, \bar{\xi}=\mathscr{O}(1)$, перепишем дифференциальные операторы (19) следующим образом:

$$
\begin{aligned}
-\varepsilon \Delta u^{\varepsilon}- & u_{y}^{\varepsilon} \sim-\varepsilon\left(\sum_{l=0}^{\infty}(l+1) \varepsilon^{l} \bar{\xi}^{l}\right) \sum_{k=0}^{\infty} \varepsilon^{k} \frac{\partial^{2} \theta^{k}}{\partial \eta^{2}}+\varepsilon\left(\sum_{l=0}^{\infty} \varepsilon^{l} \bar{\xi}^{l}\right) \sum_{k=0}^{\infty} \varepsilon^{k-1} \frac{\partial \theta^{k}}{\partial \bar{\xi}} \\
& -\varepsilon \sum_{j=0}^{\infty} \varepsilon^{j-2} \frac{\partial^{2} \theta^{j}}{\partial \bar{\xi}^{2}}+\sin \eta \sum_{j=0}^{\infty} \varepsilon^{j-1} \frac{\partial \theta^{j}}{\partial \bar{\xi}}-\cos \eta\left(\sum_{l=0}^{\infty} \varepsilon^{l} \bar{\xi}^{l}\right) \sum_{k=0}^{\infty} \varepsilon^{k} \frac{\partial \theta^{k}}{\partial \eta} \\
=- & \sum_{j=0}^{\infty} \varepsilon^{j+1}\left[\sum_{k=0}^{j}(j-k+1) \bar{\xi}^{j-k} \frac{\partial^{2} \theta^{k}}{\partial \eta^{2}}\right]+\sum_{j=0}^{\infty} \varepsilon^{j}\left[\sum_{k=0}^{j} \bar{\xi}^{j-k} \frac{\partial \theta^{k}}{\partial \bar{\xi}}\right] \\
& -\sum_{j=0}^{\infty} \varepsilon^{j-1} \frac{\partial^{2} \theta^{j}}{\partial \bar{\xi}^{2}}+\sin \eta \sum_{j=0}^{\infty} \varepsilon^{j-1} \frac{\partial \theta^{j}}{\partial \bar{\xi}}-\cos \eta \sum_{j=0}^{\infty} \varepsilon^{j}\left[\sum_{k=0}^{j} \bar{\xi}^{j-k} \frac{\partial \theta^{k}}{\partial \eta}\right]=0 .
\end{aligned}
$$


Приравнивая члены при каждом $\varepsilon^{j}$ с $\bar{\xi}=\xi / \varepsilon, \bar{\xi}=\mathscr{O}(1)$, получим, что для $0<\bar{\xi}<\infty, \pi<\eta<2 \pi, j=0,1, \ldots$

$$
\left\{\begin{array}{l}
-\frac{\partial^{2} \theta^{j}}{\partial \bar{\xi}^{2}}+\sin \eta \frac{\partial \theta^{j}}{\partial \bar{\xi}}=\sum_{k=0}^{j-2}(j-k-1) \bar{\xi}^{j-k-2} \frac{\partial^{2} \theta^{k}}{\partial \eta^{2}} \\
\quad-\sum_{k=0}^{j-1} \bar{\xi}^{j-k-1} \frac{\partial \theta^{k}}{\partial \bar{\xi}}+\cos \eta \sum_{k=0}^{j-1} \bar{\xi}^{j-k-1} \frac{\partial \theta^{k}}{\partial \eta}, \\
\theta^{j}=v^{j}(\eta):=-u^{j}(\cos \eta, \sin \eta) \quad \text { в } \bar{\xi}=0, \\
\theta^{j} \rightarrow 0 \quad \text { при } \bar{\xi} \rightarrow \infty .
\end{array}\right.
$$

Начиная с $\theta^{0}$, определенного в $(21)$, по индукции получим $\theta^{j}$ в виде $\theta^{j}=\theta_{h}^{j}+\theta_{p}^{j}$, где $\theta_{h}^{j}$ - решение задачи $(31)$, в которой правая часть $(31)_{1}$ заменена на 0 , а $\theta_{p}^{j}-$ решение задачи $(31)$, где $(31)_{2}$ заменено на $v^{j}=0$ при $\bar{\xi}=0$. Решения $\theta_{h}^{j}$ для каждого $j$ легко находятся, так же как и для $\theta^{0}$ :

$$
\theta_{h}^{j}=v^{j}(\eta) \exp ((\sin \eta) \bar{\xi}) \chi_{[\pi, 2 \pi]}(\eta) \quad \text { при } 0 \leqslant \bar{\xi}<\infty,
$$

где $v^{j}(\eta)=-u^{j}(\cos \eta, \sin \eta)$. Получая частное решение $\theta_{p}^{j}$ уравнения (32), находим по индукции, что корректоры $\theta^{j}=\theta_{h}^{j}+\theta_{p}^{j}$, как доказано в [24], имеют следующий вид.

Лемма 4. Корректоры пограничного слоя $\theta^{j}$ имеют вид

$$
\theta^{j}=P^{j}(\eta, \bar{\xi}) \exp ((\sin \eta) \bar{\xi}) \chi_{[\pi, 2 \pi]}(\eta), \quad j \geqslant 0,
$$

гдe

$$
\begin{aligned}
& P^{j}(\eta, \bar{\xi})=\sum_{i=0}^{j} \sum_{\substack{k=0 \\
a_{i, q}(\eta)}}^{2 j-2 i} a_{i, 3 j-3 i-k}(\eta) \bar{\xi}^{k}, \\
& \sum_{\substack{m+r \leqslant q \\
m, r \geqslant 0}} \frac{c_{m, r}}{\sin ^{m} \eta} \frac{d^{r} v^{i}(\eta)}{d \eta^{r}}
\end{aligned}
$$

$u v^{j}(\eta)=-u^{j}(\cos \eta, \sin \eta)$. Здесъ коэффициенты $c_{m, r}=c_{m, r}(\eta) \in \mathscr{C}^{\infty}([0,2 \pi))$ могут быть различными в различных случаях, а $\chi_{A}(\eta)$ - характеристическая функиия множества $A$.

Используем, подходящим образом, приближенную форму функций пограничного слоя $\theta^{j}$, а именно:

$$
\bar{\theta}^{j}=\theta^{j} \delta(\xi)=P^{j}(\eta, \bar{\xi}) \exp ((\sin \eta) \bar{\xi}) \delta(\xi) \chi_{[\pi, 2 \pi]}(\eta),
$$

где $\delta(\xi)$ - снова гладкая обрезывающая функция, $0 \leqslant \delta \leqslant 1$, такая, что $\delta(\xi)=1$ при $0 \leqslant \xi \leqslant 1 / 4$ и $\delta(\xi)=0$ при $\xi \geqslant 1 / 2$.

Следующие результаты о сходимости получены в [24].

Теорема 3. Пусть $u^{\varepsilon}$ - решение задачи (1), а $u_{\varepsilon n}=\sum_{j=0}^{n} \varepsilon^{j} u^{j}$, и пусть $\bar{\theta}_{\varepsilon n}=\sum_{j=0}^{n} \varepsilon^{j} \bar{\theta}^{j}$, где $u^{j}-$ решения уравнения (4), а $\bar{\theta}^{j}$ - корректоры, определенные в (36). Предположим, что выполняются условия совместности (16) 
c $\mu=1,2, j=n$, m.e.

$$
\frac{\partial^{p+q} f}{\partial x^{p} \partial y^{q}}=0 \text { в }( \pm 1,0), \quad 0 \leqslant 2 p+q \leqslant \mu+3 n, p, q, n \geqslant 0, \text { для } \mu=1 \text { или } 2 .
$$

Тогда можно установить следующую оценку:

$$
\left\|u^{\varepsilon}-u_{\varepsilon n}-\bar{\theta}_{\varepsilon n}\right\|_{\varepsilon} \leqslant \kappa \varepsilon^{n+(2+\mu) / 4} .
$$

\section{3. Случай общих тейлоровских мономов (следуя [25])}

Если $f$ несовместна, т. е. не удовлетворяет гипотезам типа $(25),(28),(37)$, представим ее в виде $\widetilde{f}+\widehat{f}$, где $\widehat{f}$ - сумма тейлоровских разложений $f$ в $( \pm 1,0)$ подходящего порядка $\mu=0,1$ или 2 :

$$
\widehat{f}=\sum_{0 \leqslant 2 p+q \leqslant \mu+3 n}\left[\frac{\partial^{p+q} f}{\partial x^{p} \partial y^{q}}(-1,0) \frac{(1-x)}{2^{p+1} p ! q !}-\frac{\partial^{p+q} f}{\partial x^{p} \partial y^{q}}(1,0) \frac{(1+x)}{(-2)^{p+1} p ! q !}\right] C_{u}(x)^{2 p} y^{q} .
$$

Заметим, что функция $\tilde{f}=f-\widehat{f}$ с $(\mu=0, n=0),(\mu=1, n=0)$ и $(\mu=2$, $n \geqslant 0)$ соответственно удовлетворяет условиям совместности (25), (28) и (37), а теоремы 1, 2 и 3 применимы с заменой $f$ на $\widetilde{f}$. Вследствие линейности осталось рассмотреть полином $\widehat{f}$, который является линейной комбинацией мономов $(1 \pm x) C_{u}(x)^{2 p} y^{q}$. Без ограничения общности рассуждений, используя симметрию и производя суперпозицию решений, достаточно рассмотреть $\widehat{f}=(1+x) C_{u}(x)^{2 p} y^{q}$, и, таким образом, осталось рассмотреть задачу

$$
\begin{cases}L_{\varepsilon} u^{\varepsilon}=\widehat{f}=(1+x) C_{u}(x)^{2 p} y^{q} & \text { в } D \\ u^{\varepsilon}=0 & \text { на } \partial D .\end{cases}
$$

Тогда получим внешнее решение нулевого порядка:

$$
u^{0}(x, y)=\frac{1}{q+1}(1+x) C_{u}(x)^{2 p}\left(C_{u}(x)^{q+1}-y^{q+1}\right) .
$$

Решение и его производные легко оценить. Оно регулярно по $y$. Если $q-$ нечетное число, то $u^{0}$ - полином по $x$ и $y$ и, следовательно, является гладким по $x, y$. Более того, из (41) следует, что $u^{0}=0$ на $\partial D$, так что нет расхождения граничных значений между $u^{\varepsilon}$ и $u^{0}$ (см. теорему 4 и замечание 1 ). Когда $q-$ четное число, легко находим, что производные $u^{0}$ имеют особенности вблизи $x= \pm 1$. Например, так как $C_{u}(x)^{\prime}=-x C_{u}(x)^{-1}$ около $x=1$, то в случае $p=q=0$

$$
u_{x}^{0}=\mathscr{O}\left((1-x)^{-1 / 2}\right), \quad u_{x x}^{0}=\mathscr{O}\left((1-x)^{-3 / 2}\right) .
$$

Если же $p=0, q=1$, то $u^{0}$ регулярна во всей области.

Для того чтобы изолировать особенности $u^{j}$ в $x= \pm 1, y=0$, мы вводим гладкую обрезывающую функцию $\rho=\rho(x) \in \mathscr{C}^{\infty}(\mathbb{R}), \rho \geqslant 0, \rho=0$ при $x \geqslant 1$, $\rho=1$ при $x \leqslant 1-\widetilde{\sigma}$, где $0<\widetilde{\sigma}<1$, и $0 \leqslant \rho(x) \leqslant 1$ при $1-\widetilde{\sigma} \leqslant x \leqslant 1$. Более 


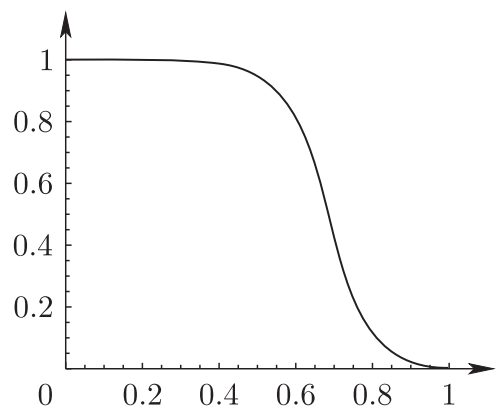

Рис. 1. График функции $е \psi(x)=e \psi_{1}(x)=\exp \left[1-\left(1-\exp \left(1-x^{-2}\right)\right)^{-1}\right]$

того, при $m \geqslant 1$ требуется, чтобы производные функции $\rho$ имели следующие свойства:

$$
\begin{aligned}
& \left|\frac{d^{m} \rho}{d x^{m}}(x)\right| \leqslant \frac{\kappa}{\tilde{\sigma}^{m}} \psi\left(\frac{x-1+\widetilde{\sigma}}{\tilde{\sigma}}\right) \chi_{(1-\widetilde{\sigma}, 1)}(x) \quad \text { для некоторого } \quad \kappa=\kappa_{m}>0, \\
& \psi(x)=\psi_{c}(x)=\exp \left[-\frac{c}{1-\exp \left(1-1 / x^{2}\right)}\right] \quad \text { для некоторого } \quad c>0
\end{aligned}
$$

(см. рис. 1); например, для $c=1$ мы можем выбрать

$$
\rho= \begin{cases}e \psi_{1}\left(\frac{x-1+\tilde{\sigma}}{\widetilde{\sigma}}\right), & \text { если } 1-\tilde{\sigma}<x<1, \\ 0, & \text { если } x \geqslant 1, \\ 1, & \text { если } x \leqslant 1-\tilde{\sigma} ;\end{cases}
$$

здесь $\widetilde{\sigma}=1-\cos \sigma$, где $\sigma>0$ мало и будет точно определено и выражено в терминах $\varepsilon$ позже (см. рис. 2). Заметим, что при малых $\sigma>0$

$$
c_{0} \sigma^{2} \leqslant \widetilde{\sigma}=1-\cos \sigma \leqslant c_{1} \sigma^{2} \text { для некоторых } c_{0}, c_{1}>0 .
$$

Разложим $\widehat{f}$ в сумму функции $f^{*}$, тождественно обращающейся в нуль в окрестности точек $( \pm 1,0)$, и функции $\widehat{f}-f^{*}$, имеющей носитель около этих точек. Таким образом, как и в [25], мы рассматриваем задачу

$$
\begin{cases}L_{\varepsilon} u^{\varepsilon}=f^{*}:=\rho(x) \check{\rho}(x)(1+x) C_{u}(x)^{2 p} y^{q} & \text { в } D, \\ u^{\varepsilon}=0 & \text { на } \partial D,\end{cases}
$$

где функции $\rho(x)$ и $\check{\rho}(x)=\rho(-x)$ из $\mathscr{C}^{\infty}(\mathbb{R})$ - гладкие обрезывающие функции из (43)-(45).

Рассмотрим уравнения для внешнего разложения функции $f^{*}$ (ср. с (4)):

$$
\begin{cases}-u_{y}^{* 0}=f^{*}=\rho(x) \check{\rho}(x)(1+x) C_{u}(x)^{2 p} y^{q} & \text { в } D, \\ u^{* 0}=0 & \text { на } \Gamma_{u}\end{cases}
$$

и для $j \geqslant 1$

$$
\begin{cases}-u_{y}^{* j}=\Delta u^{*(j-1)} & \text { в } D \\ u^{* j}=0 & \text { на } \Gamma_{u}\end{cases}
$$




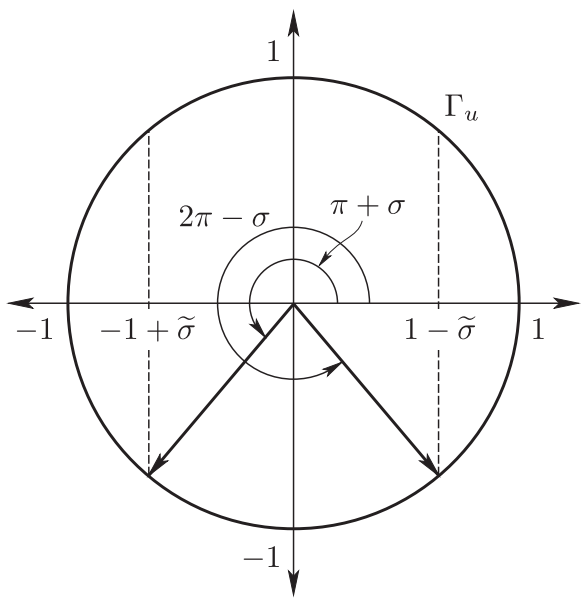

Рис. 2. Построение обрезывающей функции $\rho$ для изоляции особенностей внешних решений $u^{j}$ вблизи характеристических точек $x= \pm 1$; здесь $\cos \sigma=1-\widetilde{\sigma}$

Заметим, что $u^{* 0}=\rho \check{\rho} u^{0}$, где $u^{0}$ из (41), откуда легко заключить, что $u^{* j} \in$ $\mathscr{C}^{\infty}(\bar{D})$ для всех $j \geqslant 0$. Внешние решения $u^{* j}$ находятся аналогично (4) и (5). Для $j \geqslant 0$, таким образом, имеем

$$
u^{* j}(x, y)=\int_{y}^{C_{u}(x)} \Delta u^{*, j-1}(x, s) d s,
$$

где $\Delta u^{*,-1}=f^{*}$.

В [25] доказаны следующие свойства регулярности $u^{* j}$.

Лемма 5. Для всех $i, j, m \geqslant 0$ имеют место оценки: $n р и(x, y) \in \bar{D}$

$$
\left|\frac{\partial^{i+m} u^{* j}}{\partial x^{i} \partial y^{m}}(x, y)\right| \leqslant \kappa C_{u}(x)^{\lambda_{1}} H(x),
$$

¿де

$$
\lambda_{1}=\lambda_{1}(j, i, m)=1-3 j-2 i-m+2 p+q
$$

$u$

$$
\begin{aligned}
H(x)=\psi & \left(\frac{x-1+\widetilde{\sigma}}{\widetilde{\sigma}}\right) \chi_{(1-\widetilde{\sigma}, 1]}(x) \\
& +\psi\left(\frac{x+1-\tilde{\sigma}}{\widetilde{\sigma}}\right) \chi_{[-1,-1+\widetilde{\sigma})}(x)+\chi_{[-1+\widetilde{\sigma}, 1-\widetilde{\sigma}]}(x)
\end{aligned}
$$

$c \psi=\psi_{c} u 3(44)$

3.1. Нулевой порядок. Заметим, что $u^{\varepsilon}-u^{0}$ в общем случае не равно нулю на $\Gamma_{l}$. Чтобы учесть это отклонение на границе $\Gamma_{l}$, введем корректоры пограничного слоя, которые определяются следующим образом; в первом 
порядке

$$
\theta^{* 0}=-u^{* 0}(\cos \eta, \sin \eta) \exp \left(\frac{\sin \eta}{\varepsilon} \xi\right) \chi_{[\pi, 2 \pi]}(\eta) .
$$

Заметим, что $\theta^{* 0}$ является решением следующего уравнения:

$$
-\varepsilon \frac{\partial^{2} \theta^{* 0}}{\partial \xi^{2}}+\sin \eta \frac{\partial \theta^{* 0}}{\partial \xi}=0
$$

Рассмотрим его приближенную форму:

$$
\bar{\theta}^{* 0}(\eta, \xi)=\theta^{* 0}(\eta, \xi) \delta(\xi) .
$$

Следующие результаты о сходимости были получены в [25].

Теорема 4. Пусть $u^{\varepsilon}$ является решением задачи (40) для $p, q \geqslant 0, q=$ $2 r+1$ - нечетное. Тогда

$$
\left\|u^{\varepsilon}-u^{0}\right\|_{\varepsilon} \leqslant \kappa \varepsilon
$$

где

$$
u^{0}(x, y)=\frac{1}{2 r+2}(1+x)\left(1-x^{2}\right)^{p}\left(\left(1-x^{2}\right)^{r+1}-y^{2(r+1)}\right) .
$$

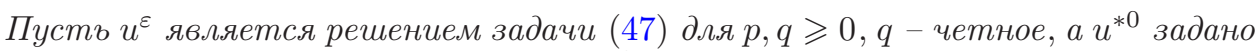
формулой (50) для $j=0$, и пусть $\bar{\theta}^{* 0}=\theta^{* 0} \delta(\xi)$ - приближенная форма $\theta^{0}$, аналогично (56). Тогда

$$
\left\|u^{\varepsilon}-u^{* 0}-\bar{\theta}^{* 0}\right\|_{\varepsilon} \leqslant \kappa \begin{cases}\varepsilon \sigma^{-1}+\min \left\{\varepsilon^{1 / 2}(-\ln \sigma)^{1 / 2}, \varepsilon \sigma^{-2}\right\}, & \text { если } p=q=0, \\ \min \left\{\varepsilon^{1 / 2}, \varepsilon(-\ln \sigma)^{1 / 2}\right\}, & \text { если } 2 p+q=2, \\ \varepsilon, & \text { если } 2 p+q \geqslant 4 .\end{cases}
$$

ЗАмечАниЕ 1 . Из соотношения (58) для $p, q \geqslant 0, q-$ нечетное, получим, что $u^{0}(\cos \eta, \sin \eta)=0$, т. е. $u^{0}=0$ на $\partial D$, и, следовательно, пограничные слои исчезают.

3.2. Высшие порядки. Будем искать улучшенные приближения $u^{\varepsilon}$, используя функции высших порядков и корректоры $u^{* j}, \theta^{* j}, \bar{\theta}^{* j}, j \geqslant 1$. Корректоры $u^{* j}$ были определены в (49); определим $\theta^{* j}, \bar{\theta}^{* j}$. Аналогично [24], введем корректоры пограничного слоя $\theta^{* j}$, соответствующие внутреннему разложению $u^{\varepsilon} \sim \sum_{j=0}^{\infty} \varepsilon^{j} \theta^{* j}$. Заметим еще раз, что

$$
(1-\xi)^{-1}=\sum_{k=0}^{\infty} \xi^{k}, \quad(1-\xi)^{-2}=\sum_{k=0}^{\infty}(k+1) \xi^{k},
$$

где $\xi=\bar{\xi} \varepsilon, \bar{\xi}=\mathscr{O}(1)$; приравнивая члены дифференциальных операторов (19) при каждой степени $\varepsilon^{j}$, для $0 \leqslant \bar{\xi}<\infty, \pi<\eta<2 \pi, j=0,1, \ldots$ получим

$$
\begin{aligned}
-\frac{\partial^{2} \theta^{* j}}{\partial \bar{\xi}^{2}}+\sin \eta \frac{\partial \theta^{* j}}{\partial \bar{\xi}}= & \sum_{k=0}^{j-2}(j-k-1) \bar{\xi}^{j-k-2} \frac{\partial^{2} \theta^{* k}}{\partial \eta^{2}} \\
& -\sum_{k=0}^{j-1} \bar{\xi}^{j-k-1} \frac{\partial \theta^{* k}}{\partial \bar{\xi}}+\cos \eta \sum_{k=0}^{j-1} \bar{\xi}^{j-k-1} \frac{\partial \theta^{* k}}{\partial \eta} .
\end{aligned}
$$


Добавим к этим уравнениям граничные условия

$$
\begin{cases}\theta^{* j}=v^{* j}(\eta)=-u^{* j}(\cos \eta, \sin \eta) & \text { в точке } \bar{\xi}=0, \\ \theta^{* j} \rightarrow 0 & \text { при } \bar{\xi} \rightarrow \infty .\end{cases}
$$

Точные решения задачи (60), (61) известны (см. [25] и лемму 4 выше) и имеют вид

$$
\theta^{* j}=P^{* j}(\eta, \bar{\xi}) \exp ((\sin \eta) \bar{\xi}) \chi_{[\pi, 2 \pi]}(\eta), \quad j \geqslant 0
$$

где

$$
\begin{aligned}
P^{* j}(\eta, \bar{\xi}) & =\sum_{i=0}^{j} \sum_{k=0}^{2 j-2 i} a_{i, 3 j-3 i-k}^{*}(\eta) \bar{\xi}^{k} \\
a_{i, q}^{*}(\eta) & =\sum_{\substack{m+r \leqslant q \\
m, r \geqslant 0}} \frac{c_{m, r}}{\sin ^{m} \eta} \frac{d^{r} v^{* i}(\eta)}{d \eta^{r}}
\end{aligned}
$$

и $v^{* j}(\eta)=-u^{* j}(\cos \eta, \sin \eta)$. Здесь $c_{m, r}=c_{m, r}(\eta) \in \mathscr{C}^{\infty}([0,2 \pi))$.

Анализ, касающийся $f^{*}=\rho(x) \check{\rho}(x)(1+x) C_{u}(x)^{2 p} y^{q}$, полностью развит в любом порядке в [25], и там же доказана следующая теорема.

ТеОРема 5. Пусть и является решением задачи (47) при $p, q \geqslant 0$, и пусть

$$
u_{\varepsilon n}^{*}=\sum_{j=0}^{n} \varepsilon^{j} u^{* j}, \quad \bar{\theta}_{\varepsilon n}^{*}=\sum_{j=0}^{n} \varepsilon^{j} \bar{\theta}^{* j},
$$

где $u^{* j}$ заданы в (50) и $\bar{\theta}^{* j}=\theta^{* j} \delta(\xi)$ являются приближенной формой $\theta^{* j}$, аналогично (62). Тогда имеем:

$$
\begin{aligned}
& \left\|u^{\varepsilon}-u_{\varepsilon n}^{*}-\bar{\theta}_{\varepsilon n}^{*}\right\|_{\varepsilon} \\
& \leqslant \kappa \varepsilon^{n+1} \begin{cases}\sigma^{-3 n+2 p+q}\left(\sigma^{-1}+\min \left\{\varepsilon^{-1 / 2}, \sigma^{-2}\right\}\right), & \text { если } 2 p+q \leqslant 3 n-1, \\
\sigma^{-1}+\min \left\{\varepsilon^{-1 / 2}(-\ln \sigma)^{1 / 2}, \sigma^{-2}\right\}, & \text { если } 2 p+q=3 n, \\
(-\ln \sigma)^{1 / 2}+\min \left\{\varepsilon^{-1 / 2}, \sigma^{-1}\right\}, & \text { если } 2 p+q=3 n+1, \\
\min \left\{\varepsilon^{-1 / 2},(-\ln \sigma)^{1 / 2}\right\}, & \text { если } 2 p+q=3 n+2, \\
1, & \text { если } 2 p+q \geqslant 3 n+3 .\end{cases}
\end{aligned}
$$

Далее в теореме 7 и разделе 6 мы покажем, как наилучшим образом использовать эти оценки, выбирая в качестве $\sigma$ наиболее подходящую степень $\varepsilon$.

\section{4. Параболические пограничные слои в характеристических точках}

Осталось рассмотреть сингулярно возмущенные задачи в виде

$$
\begin{cases}L_{\varepsilon} u^{\varepsilon}=\widehat{f}-f^{*}=(1-\rho(x) \check{\rho}(x))(1+x) C_{u}(x)^{2 p} y^{q} & \text { в } D, \\ u^{\varepsilon}=0 & \text { на } \partial D .\end{cases}
$$

Заметим, что разность $\widehat{f}-f^{*}$ имеет, как функция от $x$, компактный носитель в области $x \in[-1,-1+\widetilde{\sigma}] \cup[1-\widetilde{\sigma}, 1]$. 
Так как $0<\sigma \ll 1,1-\rho(x) \check{\rho}(x)=(1-\rho(x))+(1-\check{\rho}(x))$, необходимо только рассмотреть следующую задачу около $x=1$ :

$$
\begin{cases}L_{\varepsilon} u^{\varepsilon}=f^{* *}:=(1-\rho(x))(1+x) C_{u}(x)^{2 p} y^{q} & \text { в } D, \\ u^{\varepsilon}=0 & \text { на } \partial D .\end{cases}
$$

Для $f^{* *}=(1-\check{\rho}(x))(1+x) C_{u}(x)^{2 p} y^{q}$ аналогичный анализ выполняется около $x=-1$.

В [13] предложено ввести растянутые переменные $\left(\widehat{\xi}=\xi / \varepsilon^{2 / 3}, \widehat{\eta}=\eta / \varepsilon^{1 / 3}\right)$ и $(\bar{\xi}=\xi / \varepsilon, \bar{\eta}=\eta / \varepsilon)$ в точке $(1,0)$, приводящие к появлению так называемых промежуточного и внутреннего пограничных слоев в $(1,0)$, задаваемых соответственно дифференциальными уравнениями

$$
\begin{gathered}
-\varepsilon \frac{\partial^{2} \varphi^{0}}{\partial \xi^{2}}+\eta \frac{\partial \varphi^{0}}{\partial \xi}-\frac{\partial \varphi^{0}}{\partial \eta}=0 \\
-\varepsilon \frac{\partial^{2} \widetilde{\varphi}^{0}}{\partial \eta^{2}}-\varepsilon \frac{\partial^{2} \widetilde{\varphi}^{0}}{\partial \xi^{2}}-\frac{\partial \widetilde{\varphi}^{0}}{\partial \eta}=0 .
\end{gathered}
$$

Тем не менее наш анализ отличается от анализа в [13], и оказывается, что в нашем случае необходимо рассматривать только промежуточные (параболические) пограничные слои (68), эти пограничные слои будут обсуждаться далее в этом разделе.

Заметим, что функция $f^{* *}$ имеет носитель в области размера $\mathscr{O}(\widetilde{\sigma})=\mathscr{O}\left(\sigma^{2}\right)$ вблизи $x=1$. Легко видеть, что в граничных выпрямляющих координатах $(\eta, \xi)$ носитель $f^{* *}$ сосредоточен в области размера $\mathscr{O}\left(\sigma^{2}\right)$ по $\xi$ вблизи $\xi=0$ и в области размера $\mathscr{O}(\sigma)$ по $\eta$ вблизи $\eta=0$.

Положим

$$
g(\eta, \xi)=f^{* *}((1-\xi) \cos \eta,(1-\xi) \sin \eta) .
$$

Сначала оценим $g=g(\eta, \xi)$ в следующей лемме.

Лемма 6. Для $m, s \geqslant 0$ существует постоянная $\kappa=\kappa_{m, s}>0$, не зависящая от $\varepsilon, \sigma$, такая, что поточечно

$$
\left|\frac{\partial^{s+m} g}{\partial \xi^{s} \partial \eta^{m}}(\eta, \xi)\right| \leqslant \kappa \sigma^{2 p+q-2 s-m} \chi_{[-\sigma, \sigma] \times[0, \widetilde{\sigma}]}(\eta, \xi) .
$$

ДокАЗАТЕЛЬство. Заметим, что $g(\eta, \xi)$ - гладкая $\left(\mathscr{C}^{\infty}\right)$ и имеет носитель, сосредоточенный в $(\eta, \xi) \in[-\sigma, \sigma] \times[0, \widetilde{\sigma}]$. Из $(70)$ и $(67)$ видим, что $g(\eta, \xi)=(1-\rho((1-\xi) \cos \eta))(1+(1-\xi) \cos \eta)\left(\sin ^{2} \eta+\xi(2-\xi) \cos ^{2} \eta\right)^{p}((1-\xi) \sin \eta)^{q}$

Следовательно, раскладывая член в степени $p$, можем записать

$$
g(\eta, \xi)=\sum_{r=0}^{p} C_{r}(\eta, \xi) \xi^{p-r}(\sin \eta)^{2 r+q}
$$

где

$$
\left|\frac{\partial^{s_{1}+m_{1}}}{\partial \xi^{s_{1}} \partial \eta^{m_{1}}} C_{r}(\eta, \xi)\right| \leqslant \kappa \widetilde{\sigma}^{-s_{1}-m_{1}} \sigma^{m_{1}} \chi_{[-\sigma, \sigma] \times[0, \widetilde{\sigma}]}(\eta, \xi) .
$$


Дифференцируя $g$, находим, что

$$
\begin{aligned}
\left|\frac{\partial^{s+m} g}{\partial \xi^{s} \partial \eta^{m}}(\eta, \xi)\right| & \leqslant \kappa\left|\sum_{r=0}^{p} \sum_{\substack{0 \leqslant s_{1}+s_{2} \leqslant s \\
0 \leqslant m_{1}+m_{2} \leqslant m}} \frac{\partial^{s_{1}+m_{1}}}{\partial \xi^{s_{1}} \partial \eta^{m_{1}}} C_{r}(\eta, \xi) \xi^{p-r-s_{2}}(\sin \eta)^{2 r+q-m_{2}}\right| \\
& \leqslant \kappa \sum_{r=0}^{p} \sum_{\substack{0 \leqslant s_{1}+s_{2} \leqslant s, 0 \leqslant m_{1}+m_{2} \leqslant m}} \tilde{\sigma}^{p-r-s_{2}-s_{1}-m_{1}} \sigma^{2 r+q-m_{2}+m_{1}} \chi_{[-\sigma, \sigma] \times[0, \widetilde{\sigma}]}(\eta, \xi) \\
& \leqslant \kappa \sigma^{2 p+q-2 s-m} \chi_{[-\sigma, \sigma] \times[0, \widetilde{\sigma}]}(\eta, \xi) .
\end{aligned}
$$

Из (72) следует, что функция $g$ имеет компактный носитель в области $(\eta, \xi) \in$ $[-\sigma, \sigma] \times[0, \widetilde{\sigma}]$ и является бесконечно гладкой. Отсюда получаем, что

$$
\frac{\partial^{k} g}{\partial \eta^{k}}(\eta, \xi)=0 \quad \text { в } \eta=\sigma \text { для всех } k \geqslant 0 .
$$

Введем следующие растянутые переменные:

$$
\widetilde{\xi}=\frac{\xi}{\sigma^{2}}, \quad \widetilde{\eta}=\frac{\eta}{\sigma},
$$

где $0<\sigma \ll 1$ сравнимо со степенью величины $\varepsilon$, определенной позже.

Используя граничные выпрямляющие координаты (см. (19)) и растянутые переменные $\widetilde{\xi}, \widetilde{\eta}$, определенные в (77), преобразуем $L^{\varepsilon} u^{\varepsilon}$ из (67):

$$
-\frac{\varepsilon \sigma^{-2}}{(1-\xi)^{2}} \frac{\partial^{2} u^{\varepsilon}}{\partial \widetilde{\eta}^{2}}+\frac{\varepsilon \sigma^{-2}}{1-\xi} \frac{\partial u^{\varepsilon}}{\partial \widetilde{\xi}}-\varepsilon \sigma^{-4} \frac{\partial^{2} u^{\varepsilon}}{\partial \widetilde{\xi}^{2}}+\sigma^{-2} \sin \eta \frac{\partial u^{\varepsilon}}{\partial \widetilde{\xi}}-\sigma^{-1} \frac{\cos \eta}{1-\xi} \frac{\partial u^{\varepsilon}}{\partial \widetilde{\eta}} .
$$

Так как $\sin \eta=\mathscr{O}(\sigma)$, а $1-\cos \eta=\mathscr{O}\left(\sigma^{2}\right)$ на носителе $g$, то $\sin (\eta) \sigma^{-2}$ и $(1-\cos \eta) \sigma^{-3}$ сравнимы с $\sigma^{-1}$ на носителе $g$; замечая также, что $\varepsilon \sigma^{-2} \ll \varepsilon \sigma^{-4}$, и записывая $\cos \eta=1-(1-\cos \eta)$, находим, что старшие члены (78) имеют вид

$$
-\varepsilon \sigma^{-4} \frac{\partial^{2} u^{\varepsilon}}{\partial \widetilde{\xi}^{2}}+\sigma^{-2} \sin \eta \frac{\partial u^{\varepsilon}}{\partial \widetilde{\xi}}-\sigma^{-1} \frac{\partial u^{\varepsilon}}{\partial \widetilde{\eta}} .
$$

Затем мы подставляем формальное разложение

$$
u^{\varepsilon} \sim \sum_{j=0}^{\infty} \sigma^{j} \varphi^{j}
$$

и используем в (78) соотношения

$$
\begin{aligned}
& (1-\xi)^{-1}=\sum_{k=0}^{\infty} \xi^{k}=\sum_{k=0}^{\infty} \sigma^{2 k} \widetilde{\xi}^{k}, \\
& (1-\xi)^{-2}=\sum_{k=0}^{\infty}(k+1) \xi^{k}=\sum_{k=0}^{\infty}(k+1) \sigma^{2 k} \widetilde{\xi}^{k},
\end{aligned}
$$

где $\widetilde{\xi}$ определено в (77). Отсюда формально получим, что

$$
\begin{aligned}
-\varepsilon \sigma^{-4} \sum_{j=0}^{\infty} & \sum_{k=0}^{\infty}(k+1) \sigma^{2 k+j+2} \widetilde{\xi}^{k} \frac{\partial^{2} \varphi^{j}}{\partial \widetilde{\eta}^{2}}+\varepsilon \sigma^{-4} \sum_{j=0}^{\infty} \sum_{k=0}^{\infty} \sigma^{2 k+j+2} \widetilde{\xi}^{k} \frac{\partial \varphi^{j}}{\partial \widetilde{\xi}} \\
& -\varepsilon \sigma^{-4} \sum_{j=0}^{\infty} \sigma^{j} \frac{\partial^{2} \varphi^{j}}{\partial \widetilde{\xi}^{2}}+\sigma^{-2} \eta \sum_{j=0}^{\infty} \sigma^{j} \frac{\partial \varphi^{j}}{\partial \widetilde{\xi}}+\sigma^{-4}(\sin \eta-\eta) \sum_{j=0}^{\infty} \sigma^{j+2} \frac{\partial \varphi^{j}}{\partial \widetilde{\xi}}
\end{aligned}
$$




$$
\begin{aligned}
& \quad-\sigma^{-1} \sum_{j=0}^{\infty} \sum_{k=0}^{\infty} \sigma^{2 k+j} \widetilde{\xi}^{k} \frac{\partial \varphi^{j}}{\partial \widetilde{\eta}}+\sigma^{-3}(1-\cos \eta) \sum_{j=0}^{\infty} \sum_{k=0}^{\infty} \sigma^{2 k+j+2} \widetilde{\xi}^{k} \frac{\partial \varphi^{j}}{\partial \widetilde{\eta}} \\
& =g(\eta, \xi) .
\end{aligned}
$$

Так как $g(\eta, \xi)=\mathscr{O}\left(\sigma^{2 p+q}\right)($ см. $(71))$, в старшем порядке имеем: для $j=2 p+q$

$$
-\varepsilon \sigma^{-4} \frac{\partial^{2} \varphi^{j}}{\partial \widetilde{\xi}^{2}}+\sigma^{-1} \widetilde{\eta} \frac{\partial \varphi^{j}}{\partial \widetilde{\xi}}-\sigma^{-1} \frac{\partial \varphi^{j}}{\partial \widetilde{\eta}}=g(\eta, \xi),
$$

а для $j \geqslant 2 p+q+1$

$$
\begin{gathered}
-\varepsilon \sigma^{-4} \frac{\partial^{2} \varphi^{j}}{\partial \widetilde{\xi}^{2}}+\sigma^{-1} \widetilde{\eta} \frac{\partial \varphi^{j}}{\partial \widetilde{\xi}}-\sigma^{-1} \frac{\partial \varphi^{j}}{\partial \widetilde{\eta}} \\
=\varepsilon \sigma^{-4} \sum_{k=0}^{\infty}(k+1) \widetilde{\xi}^{k} \frac{\partial^{2} \varphi^{j-2 k-2}}{\partial \widetilde{\eta}^{2}}-\varepsilon \sigma^{-4} \sum_{k=0}^{\infty} \widetilde{\xi}^{k} \frac{\partial \varphi^{j-2 k-2}}{\partial \widetilde{\xi}} \\
-\sigma^{-4}(\sin \eta-\eta) \frac{\partial \varphi^{j-2}}{\partial \widetilde{\xi}}+\sigma^{-1} \sum_{k=1}^{\infty} \widetilde{\xi}^{k} \frac{\partial \varphi^{j-2 k}}{\partial \widetilde{\eta}} \\
-\sigma^{-3}(1-\cos \eta) \sum_{k=0}^{\infty} \widetilde{\xi}^{k} \frac{\partial \varphi^{j-2 k-2}}{\partial \widetilde{\eta}}
\end{gathered}
$$

Здесь для удобства мы полагаем

$$
\varphi^{j}=0 \quad \text { для всех } j<2 p+q .
$$

Возвращаясь к переменным $\xi, \eta$, получим

$$
-\varepsilon \frac{\partial^{2} \varphi^{j}}{\partial \xi^{2}}+\eta \frac{\partial \varphi^{j}}{\partial \xi}-\frac{\partial \varphi^{j}}{\partial \eta}=R_{\varphi}^{j}, \quad(\eta, \xi) \in(-\infty, \sigma) \times(0,1),
$$

где

$$
\begin{aligned}
& R_{\varphi}^{j}=g(\eta, \xi) \quad \text { для } j=2 p+q, \\
& R_{\varphi}^{j}=\varepsilon \sigma^{-2} \sum_{k=0}^{\infty}(k+1)\left(\frac{\xi}{\sigma^{2}}\right)^{k} \frac{\partial^{2} \varphi^{j-2 k-2}}{\partial \eta^{2}}-\varepsilon \sigma^{-2} \sum_{k=0}^{\infty}\left(\frac{\xi}{\sigma^{2}}\right)^{k} \frac{\partial \varphi^{j-2 k-2}}{\partial \xi} \\
& \quad-\sigma^{-2}(\sin \eta-\eta) \frac{\partial \varphi^{j-2}}{\partial \xi}+\sum_{k=1}^{\infty}\left(\frac{\xi}{\sigma^{2}}\right)^{k} \frac{\partial \varphi^{j-2 k}}{\partial \eta} \\
& -\sigma^{-2}(1-\cos \eta) \sum_{k=0}^{\infty}\left(\frac{\xi}{\sigma^{2}}\right)^{k} \frac{\partial \varphi^{j-2 k-2}}{\partial \eta} \quad \text { для } j \geqslant 2 p+q+1
\end{aligned}
$$

И

$$
\text { все } \varphi^{j} \text { обращаются в нуль, если } \eta=\sigma \text { или } \xi=0,1 .
$$

Из рекуррентности видно, что (84)-(86) есть линейная параболическая задача для $\varphi^{j}$, где $\xi$ - пространственная переменная, а $\eta$ (фактически $-\eta$ ) - временная переменная. Корректная постановка для этих уравнений стандартна. 
ЗАмечАниЕ 2. Из (83)-(86) легко рекуррентно найдем, что

$$
\varphi^{j}=0, \quad \text { если } j<2 p+q \text { или } j=2 p+q+2 i+1, i \geqslant 0 .
$$

Следовательно, при $j \geqslant 2 p+q$ необходимо рассмотреть только случай, когда $j-2 p-q-$ четное.

Чтобы вывести оценки для параболических пограничных слоев $\varphi^{j}$, для краткости обозначений ниже положим $\varphi=\varphi^{j}, h=R_{\varphi}^{j}$ и рассмотрим следующую основную задачу:

$$
-\varepsilon \frac{\partial^{2} \varphi}{\partial \xi^{2}}+\eta \frac{\partial \varphi}{\partial \xi}-\frac{\partial \varphi}{\partial \eta}=h, \quad(\eta, \xi) \in(-\infty, \sigma) \times(0,1),
$$

где добавляются следующие условия:

$$
\varphi=0, \quad \text { если } \eta=\sigma \text { или } \xi=0,1 .
$$

$\mathrm{K}$ тому же, напомним (см. (76)), что $\frac{\partial^{k} g}{\partial \eta^{k}}(\eta, \xi)=0$ при $\eta=\sigma$ для всех $k \geqslant 0$. Дифференцируя (84) по $\eta$ при $j=2 p+q$ и используя (86), находим, что $\frac{\partial^{k} \varphi^{j}}{\partial \eta^{k}}(\eta, \xi)=0$ при $\eta=\sigma$ для всех $k \geqslant 0, j=2 p+q$. Из (86) снова найдем рекуррентно, что это выполняется для $R_{\varphi}^{j}$ при $j>2 p+q$ и, значит, для $\varphi^{j}$ при $j>2 p+q$. Следовательно, мы можем считать, что в (88), (89) для всех $k \geqslant 0$

$$
\frac{\partial^{k} \varphi}{\partial \eta^{k}}(\eta, \xi)=0, \quad \text { если } \eta=\sigma \text { или } \xi=0,1 .
$$

Нам будет полезна следующая лемма.

ЛЕмма 7. Для интегрируемой функиии $v=v(\eta, \xi) \geqslant 0$ u $r \leqslant \sigma$ существует постоянная $\kappa>0$, не зависящая от $\sigma, \varepsilon$, такая, что

$$
\sigma^{-1} \int_{0}^{1} \int_{r}^{\sigma} v d \eta d \xi \leqslant 2 \sup _{\eta} \int_{0}^{1} v d \xi+\frac{1}{2} \int_{0}^{1} \int_{r}^{\sigma}\left(\frac{\sigma-\eta}{\sigma^{2}}\right) v d \eta d \xi .
$$

ДокАЗАТЕЛЬСтво. Для $r<-\sigma$, разбивая на части область интегрирования, имеем

$$
\begin{array}{r}
\sigma^{-1} \int_{0}^{1} \int_{r}^{\sigma} v d \eta d \xi=\sigma^{-1} \int_{0}^{1} \int_{-\sigma}^{\sigma} v d \eta d \xi \\
+\sigma^{-1} \int_{0}^{1} \int_{r}^{-\sigma}\left(\frac{\sigma^{2}}{\sigma-\eta}\right)\left(\frac{\sigma-\eta}{\sigma^{2}}\right) v d \eta d \xi \\
\leqslant 2 \sup _{\eta} \int_{0}^{1} v d \xi+\frac{1}{2} \int_{0}^{1} \int_{r}^{-\sigma}\left(\frac{\sigma-\eta}{\sigma^{2}}\right) v d \eta d \xi
\end{array}
$$

Для $-\sigma \leqslant r \leqslant \sigma$ левая часть в $(91)$ ограничена величиной

$$
\sigma^{-1} \int_{0}^{1} \int_{-\sigma}^{\sigma} v d \eta d \xi \leqslant 2 \sup _{\eta} \int_{0}^{1} v d \xi
$$


Для простоты последующего анализа будем считать далее, что

$$
0<\varepsilon \sigma^{-3} \leqslant 1, \quad \text { т. е. } 0<\varepsilon \leqslant \sigma^{3} .
$$

Докажем сначала следующую лемму, полезную для получения оценок, которым подчиняются параболические пограничные слои и их производные.

Лемма 8. Предположим, что выполнено условие (93), т.е. $0<\varepsilon \sigma^{-3} \leqslant 1$. $\Pi$ Путь

$$
\Phi=\Phi^{m}=\frac{\partial^{m} \varphi}{\partial \eta^{m}}, \quad H=H^{m}=\frac{\partial^{m} h}{\partial \eta^{m}}, \quad D^{\prime}=(-\infty, \sigma) \times(0,1),
$$

где ч и h такие, как в уравнении (88), и рассмотрим керн-функиию:

$$
M_{l, d}=M_{l, d}(\eta, \xi)=\left(\frac{\sigma-\eta}{\sigma}\right)^{l}\left(\frac{\xi}{\sigma^{2}}\right)^{d}, \quad l, d \geqslant 0, \quad(\eta, \xi) \in D^{\prime} .
$$

Тогда существует постоянная $\kappa>0$, не зависящая от $\sigma, \varepsilon$, такая, что

$$
\begin{gathered}
\varepsilon \int_{D^{\prime}} M_{l, d}\left|\frac{\partial \Phi}{\partial \xi}\right|^{2} d \eta d \xi+d \sigma^{-1} \int_{D^{\prime}} M_{l, d-1}|\Phi|^{2} d \eta d \xi \\
\leqslant \kappa \sum_{m^{\prime}=0}^{m}\left(\varepsilon^{-1} \sigma\right)^{m-m^{\prime}}\left(C\left(m^{\prime}\right)\right)^{2}
\end{gathered}
$$

¿əe

$$
\begin{gathered}
C\left(m^{\prime}\right)=\sum_{s=0}^{m^{\prime}} \int_{-\infty}^{\sigma}\left[\int_{0}^{1} \mathscr{M}_{l, d}\left|H^{s}\right|^{2} d \xi\right]^{1 / 2} d \eta, \\
\mathscr{M}_{l, d}=1+M_{l, 0}+M_{0, d}+M_{l, d} .
\end{gathered}
$$

ДокАзАтельство. Докажем, что

$$
\begin{gathered}
\varepsilon \int_{D^{\prime}} M_{l, d}\left|\frac{\partial \Phi}{\partial \xi}\right|^{2} d \eta d \xi+d \int_{D^{\prime}}\left(\frac{\sigma-\eta}{\sigma^{2}}\right) M_{l, d-1}|\Phi|^{2} d \eta d \xi \\
+\sup _{\eta} \int_{0}^{1} M_{l, d}|\Phi(\eta, \xi)|^{2} d \xi \\
\leqslant \kappa \sum_{m^{\prime}=0}^{m}\left(\varepsilon^{-1} \sigma\right)^{m-m^{\prime}}\left(C\left(m^{\prime}\right)\right)^{2}
\end{gathered}
$$

что, как следует из леммы 7, влечет за собой (96).

Пусть $n=l+d \geqslant 0$. Для того чтобы доказать (99), выполним двойную индукцию по $m$ и $n$.

А) Рассмотрим порядок $m=0$ (т. е. $\Phi=\varphi$ ).

i) Случай $n=0$ (т.е. $l=d=0$ ). Умножая (88) на $\varphi$ и интегрируя по переменным $\eta$ и $\xi$ по области $(r, \sigma) \times(0,1), r \leqslant \sigma$, находим, используя граничные условия (89):

$$
\begin{aligned}
& \varepsilon \int_{0}^{1} \int_{r}^{\sigma}\left|\frac{\partial \varphi}{\partial \xi}\right|^{2} d \eta d \xi+\frac{1}{2} \int_{0}^{1}|\varphi(r, \xi)|^{2} d \xi \\
& \leqslant \int_{0}^{1} \int_{r}^{\sigma}|h||\varphi| d \eta d \xi \leqslant\left[\sup _{\eta} \int_{0}^{1}|\varphi(\eta, \xi)|^{2} d \xi\right]^{1 / 2} \int_{r}^{\sigma}\left[\int_{0}^{1}|h|^{2} d \xi\right]^{1 / 2} d \eta \\
& \leqslant C(0)\left[\sup _{\eta} \int_{0}^{1}|\varphi(\eta, \xi)|^{2} d \xi\right]^{1 / 2}
\end{aligned}
$$


Беря верхнюю грань по $r$, получим, что

$$
\frac{1}{4} \sup _{\eta} \int_{0}^{1}|\varphi(\eta, \xi)|^{2} d \eta \leqslant(C(0))^{2}
$$

и, следовательно,

$$
\varepsilon \int_{0}^{1} \int_{r}^{\sigma}\left|\frac{\partial \varphi}{\partial \xi}\right|^{2} d \eta d \xi+\sup _{\eta} \int_{0}^{1}|\varphi(\eta, \xi)|^{2} d \xi \leqslant 2(C(0))^{2} .
$$

Это доказывает (99) для $n=0$.

ii) Предположим, что $n \geqslant 1$ и неравенство (99) выполнено для порядков $0 \leqslant l+d \leqslant n-1$. Для $l+d=n$ умножим (88) на $M_{l, d} \varphi$ и проинтегрируем по переменным $\eta$ и $\xi$ по области $(r, \sigma) \times(0,1)$. Если не оговорено противное, $M_{l, d}=M_{l, d}(\eta, \xi)$ определено в (95). Для каждого члена в отдельности получим:

$$
\begin{aligned}
& -\varepsilon \int_{0}^{1} \int_{r}^{\sigma} \frac{\partial^{2} \varphi}{\partial \xi^{2}} M_{l, d} \varphi d \eta d \xi=\varepsilon \int_{0}^{1} \int_{r}^{\sigma} M_{l, d}\left|\frac{\partial \varphi}{\partial \xi}\right|^{2} d \eta d \xi \\
& \quad-d(d-1) \frac{\varepsilon \sigma^{-4}}{2} \int_{0}^{1} \int_{r}^{\sigma} M_{l, d-2}|\varphi|^{2} d \eta d \xi \\
& \int_{0}^{1} \int_{r}^{\sigma} \eta \frac{\partial \varphi}{\partial \xi} M_{l, d} \varphi d \eta d \xi=-\frac{d \sigma^{-2}}{2} \int_{0}^{1} \int_{r}^{\sigma} M_{l, d-1} \eta|\varphi|^{2} d \eta d \xi \\
& \quad=\frac{d}{2} \int_{0}^{1} \int_{r}^{\sigma} M_{l, d-1}\left(\frac{\sigma-\eta}{\sigma^{2}}\right)|\varphi|^{2} d \eta d \xi-\frac{d \sigma^{-1}}{2} \int_{0}^{1} \int_{r}^{\sigma} M_{l, d-1}|\varphi|^{2} d \eta d \xi \\
& -\int_{0}^{1} \int_{r}^{\sigma} \frac{\partial \varphi}{\partial \eta} M_{l, d} \varphi d \eta d \xi=\frac{1}{2} \int_{0}^{1} M_{l, d}(r, \xi)|\varphi(r, \xi)|^{2} d \eta d \xi \\
& \quad-\frac{l \sigma^{-1}}{2} \int_{0}^{1} \int_{r}^{\sigma} M_{l-1, d}|\varphi|^{2} d \eta d \xi \\
& \left|\int_{0}^{1} \int_{r}^{\sigma} h M_{l, d} \varphi d \eta d \xi\right| \\
& \leqslant\left[\sup _{\eta}^{1} \int_{0}^{1} M_{l, d}|\varphi(\eta, \xi)|^{2} d \xi\right]_{r}^{1 / 2} \int_{r}^{\sigma}\left[\int_{0}^{1} M_{l, d}|h|^{2} d \xi\right]^{1 / 2} d \eta .
\end{aligned}
$$

Отсюда аналогично (101), (102) находим, что

$$
\begin{aligned}
\varepsilon \int_{0}^{1} \int_{r}^{\sigma} & M_{l, d}\left|\frac{\partial \varphi}{\partial \xi}\right|^{2} d \eta d \xi+\frac{d}{2} \int_{0}^{1} \int_{r}^{\sigma} M_{l, d-1}\left(\frac{\sigma-\eta}{\sigma^{2}}\right)|\varphi|^{2} d \eta d \xi \\
& +\sup _{\eta} \int_{0}^{1} M_{l, d}(\eta, \xi)|\varphi(\eta, \xi)|^{2} d \xi \\
\leqslant & d(d-1) \varepsilon \sigma^{-4} \int_{0}^{1} \int_{r}^{\sigma} M_{l, d-2}|\varphi|^{2} d \eta d \xi \\
& +\kappa d \sigma^{-1} \int_{0}^{1} \int_{r}^{\sigma} M_{l, d-1}|\varphi|^{2} d \eta d \xi \\
& +\kappa l \sigma^{-1} \int_{0}^{1} \int_{r}^{\sigma} M_{l-1, d}|\varphi|^{2} d \eta d \xi+\kappa(C(0))^{2} .
\end{aligned}
$$


Благодаря предположению индукции и условию $0<\varepsilon \sigma^{-3} \leqslant 1$, использование вышеизложенного и леммы 7 доказывает (99) в случае $m=0$ для всех $l, d \geqslant 0$. Здесь мы воспользовались тем фактом, что для всех $(\eta, \xi) \in(-\infty, \sigma) \times(0,1)$

$$
M_{l-1, d}, M_{l, d-1}, M_{l, d-2} \leqslant \kappa \mathscr{M}_{l, d}
$$

(см. (98)).

В) Для $m \geqslant 1$ и для всех $l, d \geqslant 0$ предположим, что (99) выполнено для порядков, меньших или равных $m-1$, при всех $l, d \geqslant 0$.

Дифференцируя (88) $m$ раз по $\eta$, находим, что

$$
-\varepsilon \frac{\partial^{2}}{\partial \xi^{2}}\left[\frac{\partial^{m} \varphi}{\partial \eta^{m}}\right]+m \frac{\partial}{\partial \xi}\left[\frac{\partial^{m-1} \varphi}{\partial \eta^{m-1}}\right]+\eta \frac{\partial}{\partial \xi}\left[\frac{\partial^{m} \varphi}{\partial \eta^{m}}\right]-\frac{\partial}{\partial \eta}\left[\frac{\partial^{m} \varphi}{\partial \eta^{m}}\right]=\frac{\partial^{m} h}{\partial \eta^{m}}(\eta, \xi) .
$$

Используя обозначения $\Phi=\Phi^{m}$ из $(94)$, имеем, таким образом,

$$
-\varepsilon \frac{\partial^{2} \Phi}{\partial \xi^{2}}+\eta \frac{\partial \Phi}{\partial \xi}-\frac{\partial \Phi}{\partial \eta}=H-m \frac{\partial \Phi^{m-1}}{\partial \xi} .
$$

Из (90) мы находим также, что

$$
\Phi=0, \quad \text { если } \eta=\sigma, \quad \Phi=0 \quad \text { при } \xi=0,1 .
$$

i) Случай $n=0$ (т. е. $l=d=0$ ). Умножая (109) на $\Phi$ и интегрируя по $(r, \sigma) \times(0,1)$, из леммы 7 и граничных условий $\Phi=0$ при $\xi=0,1$ заключаем, что

$$
\begin{aligned}
m \mid \int_{0}^{1} & \int_{r}^{\sigma} \frac{\partial \Phi^{m-1}}{\partial \xi} \Phi d \eta d \xi|=m| \int_{0}^{1} \int_{r}^{\sigma} \Phi^{m-1} \frac{\partial \Phi}{\partial \xi} d \eta d \xi \mid \\
\leqslant & \frac{\varepsilon^{-1} m^{2}}{2} \int_{0}^{1} \int_{r}^{\sigma}\left|\Phi^{m-1}\right|^{2} d \eta d \xi+\frac{\varepsilon}{2} \int_{0}^{1} \int_{r}^{\sigma}\left|\frac{\partial \Phi}{\partial \xi}\right|^{2} d \eta d \xi \\
\leqslant & \frac{\varepsilon^{-1} \sigma m^{2}}{2}\left\{2 \sup _{\eta} \int_{0}^{1}\left|\Phi^{m-1}\right|^{2} d \xi+\frac{1}{2} \int_{0}^{1} \int_{r}^{\sigma}\left(\frac{\sigma-\eta}{\sigma^{2}}\right)\left|\Phi^{m-1}\right|^{2} d \eta d \xi\right\} \\
& +\frac{\varepsilon}{2} \int_{0}^{1} \int_{r}^{\sigma}\left|\frac{\partial \Phi}{\partial \xi}\right|^{2} d \eta d \xi .
\end{aligned}
$$

По индукции находим, что

$$
\begin{aligned}
\frac{\varepsilon}{2} \int_{0}^{1} \int_{r}^{\sigma}\left|\frac{\partial \Phi}{\partial \xi}\right|^{2} d \eta d \xi+\frac{1}{2} \int_{0}^{1}|\Phi(r, \xi)|^{2} d \xi & {\left[\sup _{\eta} \int_{0}^{1}|\Phi(\eta, \xi)|^{2} d \xi\right]^{1 / 2} \int_{r}^{\sigma}\left[\int_{0}^{1}|H|^{2} d \xi\right]^{1 / 2} d \eta } \\
& +\frac{\varepsilon^{-1} \sigma m^{2}}{2}\left\{2 \sup _{\eta} \int_{0}^{1}\left|\Phi^{m-1}\right|^{2} d \xi+\frac{1}{2} \int_{0}^{1} \int_{r}^{\sigma}\left(\frac{\sigma-\eta}{\sigma^{2}}\right)\left|\Phi^{m-1}\right|^{2} d \eta d \xi\right\} \\
\leqslant & {\left[\sup _{\eta} \int_{0}^{1}|\Phi(\eta, \xi)|^{2} d \xi\right]^{1 / 2} \int_{r}^{\sigma}\left[\int_{0}^{1}|H|^{2} d \xi\right]^{1 / 2} d \eta } \\
& +\kappa\left(\varepsilon^{-1} \sigma\right) \sum_{m^{\prime}=0}^{m-1}\left(\varepsilon^{-1} \sigma\right)^{m-1-m^{\prime}}\left(C\left(m^{\prime}\right)\right)^{2} .
\end{aligned}
$$


Беря верхнюю грань по $r$, аналогично получим, что

$$
\varepsilon \int_{0}^{1} \int_{r}^{\sigma}\left|\frac{\partial \Phi}{\partial \xi}\right|^{2} d \eta d \xi+\sup _{\eta} \int_{0}^{1}|\Phi(\eta, \xi)|^{2} d \xi \leqslant \kappa \sum_{m^{\prime}=0}^{m}\left(\varepsilon^{-1} \sigma\right)^{m-m^{\prime}}\left(C\left(m^{\prime}\right)\right)^{2} .
$$

Это доказывает случай $n=0$.

ii) Для фиксированного $m \geqslant 1$ проведем индукцию по $n=l+d$. Предположим, что (99) выполняется для всех порядков $0 \leqslant l+d \leqslant n-1$, и докажем, что оно верно для порядка $l+d=n$.

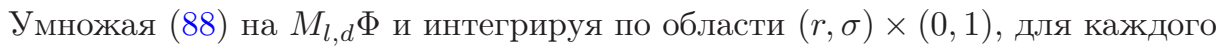
члена в отдельности получим:

$$
\begin{aligned}
& -\varepsilon \int_{0}^{1} \int_{r}^{\sigma} \frac{\partial^{2} \Phi}{\partial \xi^{2}} M_{l, d} \Phi d \eta d \xi=\varepsilon \int_{0}^{1} \int_{r}^{\sigma} M_{l, d}\left|\frac{\partial \Phi}{\partial \xi}\right|^{2} d \eta d \xi \\
& -d(d-1) \frac{\varepsilon \sigma^{-4}}{2} \int_{0}^{1} \int_{r}^{\sigma} M_{l, d-2}|\Phi|^{2} d \eta d \xi \\
& \int_{0}^{1} \int_{r}^{\sigma} \eta \frac{\partial \Phi}{\partial \xi} M_{l, d} \Phi d \eta d \xi=\frac{d}{2} \int_{0}^{1} \int_{r}^{\sigma} M_{l, d-1}\left(\frac{\sigma-\eta}{\sigma^{2}}\right)|\Phi|^{2} d \eta d \xi \\
& -\frac{d \sigma^{-1}}{2} \int_{0}^{1} \int_{r}^{\sigma} M_{l, d-1}|\Phi|^{2} d \eta d \xi \\
& -\int_{0}^{1} \quad \int_{r}^{\sigma} \frac{\partial \Phi}{\partial \eta} M_{l, d} \Phi d \eta d \xi=\frac{1}{2} \int_{0}^{1} M_{l, d}|\Phi(r, \xi)|^{2} d \xi \\
& -\frac{l \sigma^{-1}}{2} \int_{0}^{1} \int_{r}^{\sigma} M_{l-1, d}|\Phi|^{2} d \eta d \xi \\
& \left|\int_{0}^{1} \quad \int_{r}^{\sigma} H \cdot M_{l, d} \Phi d \eta d \xi\right| \\
& \leqslant\left[\sup _{\eta}^{1} M_{0} M_{l, d}|\Phi(\eta, \xi)|^{2} d \xi\right]_{r}^{1 / 2} \int_{0}^{\sigma}\left[\int_{0}^{1} M_{l, d}|H|^{2} d \xi\right]^{1 / 2} d \eta .
\end{aligned}
$$

Воспользовавшись леммой 7 , неравенством Харди и граничными условиями $\Phi=0$ при $\xi=0,1$, для последнего члена в (109) получаем:

$$
\begin{aligned}
m\left|\int_{0}^{1} \int_{r}^{\sigma} M_{l, d} \frac{\partial \Phi^{m-1}}{\partial \xi} \Phi d \eta d \xi\right| & \stackrel{(*)}{\leqslant} \kappa d\left|\int_{0}^{1} \int_{r}^{\sigma} M_{l, d} \Phi^{m-1} \xi^{-1} \Phi d \eta d \xi\right|+\kappa\left|\int_{0}^{1} \int_{r}^{\sigma} M_{l, d} \Phi^{m-1} \frac{\partial \Phi}{\partial \xi} d \eta d \xi\right| \\
\leqslant \kappa & {\left[\int_{0}^{1} \int_{r}^{\sigma} M_{2 l, 2 d}\left|\Phi^{m-1}\right|^{2} d \eta d \xi\right]^{1 / 2}\left[\int_{0}^{1} \int_{r}^{\sigma}\left|\frac{\partial \Phi}{\partial \xi}\right|^{2} d \eta d \xi\right]^{1 / 2} } \\
& +\kappa \varepsilon^{-1} \int_{0}^{1} \int_{r}^{\sigma} M_{l, d}\left|\Phi^{m-1}\right|^{2} d \eta d \xi+\frac{\varepsilon}{2} \int_{0}^{1} \int_{r}^{\sigma} M_{l, d}\left|\frac{\partial \Phi}{\partial \xi}\right|^{2} d \eta d \xi \\
\leqslant & \kappa \varepsilon \\
& \int_{0}^{1} \int_{r}^{\sigma}\left(M_{2 l, 2 d}+M_{l, d}\right)\left|\Phi^{m-1}\right|^{2} d \eta d \xi \\
& +\frac{\varepsilon}{2} \int_{0}^{1} \int_{r}^{\sigma}\left(1+M_{l, d}\right)\left|\frac{\partial \Phi}{\partial \xi}\right|^{2} d \eta d \xi
\end{aligned}
$$

3 УМН, т. 69 , вып. 3 
$\left(\right.$ в $\stackrel{(*)}{\leqslant}$ мы воспользовались равенством $\left.\frac{\partial M_{l, d}}{\partial \xi}=\frac{d}{\xi} M_{l, d}\right)$, и, значит,

$$
\begin{aligned}
m \mid \int_{0}^{1} & \int_{r}^{\sigma} M_{l, d} \frac{\partial \Phi^{m-1}}{\partial \xi} \Phi d \eta d \xi \mid \leqslant \kappa \varepsilon^{-1} \sigma\left\{\sup _{\eta} \int_{0}^{1}\left(M_{2 l, 2 d}+M_{l, d}\right)\left|\Phi^{m-1}\right|^{2} d \xi\right. \\
& \left.+\int_{0}^{1} \int_{r}^{\sigma}\left(\frac{\sigma-\eta}{\sigma^{2}}\right)\left(M_{2 l, 2 d}+M_{l, d}\right)\left|\Phi^{m-1}\right|^{2} d \eta d \xi\right\} \\
& +\frac{\varepsilon}{2} \int_{0}^{1} \int_{r}^{\sigma}\left(1+M_{l, d}\right)\left|\frac{\partial \Phi}{\partial \xi}\right|^{2} d \eta d \xi .
\end{aligned}
$$

Снова используя условие $0<\varepsilon \sigma^{-3} \leqslant 1$ и лемму 7 , заметим, что

$$
\begin{aligned}
& \frac{d \sigma^{-1}}{2} \int_{0}^{1} \int_{r}^{\sigma} M_{l, d-1}|\Phi|^{2} d \eta d \xi \\
& \quad \leqslant d \sup _{\eta} \int_{0}^{1} M_{l, d-1}|\Phi|^{2} d \xi+\frac{d}{4} \int_{0}^{1} \int_{r}^{\sigma} M_{l, d-1}\left(\frac{\sigma-\eta}{\sigma^{2}}\right)|\Phi|^{2} d \eta d \xi \\
& d(d-1) \frac{\varepsilon \sigma^{-4}}{2} \int_{0}^{1} \int_{r}^{\sigma} M_{l, d-2}|\Phi|^{2} d \eta d \xi \\
& \quad \leqslant \kappa \sup _{\eta}^{1} \int_{0}^{1} M_{l, d-2}|\Phi|^{2} d \xi+\kappa \int_{0}^{1} \int_{r}^{\sigma} M_{l, d-2}\left(\frac{\sigma-\eta}{\sigma^{2}}\right)|\Phi|^{2} d \eta d \xi \\
& \frac{l \sigma^{-1}}{2} \int_{0}^{1} \int_{r}^{\sigma} M_{l-1, d}|\Phi|^{2} d \eta d \xi \\
& \leqslant \kappa \sup _{\eta}^{1} \int_{0}^{1} M_{l-1, d}|\Phi|^{2} d \xi+\kappa \int_{0}^{1} \int_{r}^{\sigma} M_{l-1, d}\left(\frac{\sigma-\eta}{\sigma^{2}}\right)|\Phi|^{2} d \eta d \xi .
\end{aligned}
$$

Отсюда по индукции получим

$$
\begin{aligned}
\frac{\varepsilon}{2} \int_{0}^{1} \int_{r}^{\sigma} M_{l, d}\left|\frac{\partial \Phi}{\partial \xi}\right|^{2} d \eta d \xi+\frac{d}{4} \int_{0}^{1} \int_{r}^{\sigma} M_{l, d-1}\left(\frac{\sigma-\eta}{\sigma^{2}}\right)|\Phi|^{2} d \eta d \xi \\
+\frac{1}{2} \int_{0}^{1} M_{l, d}|\Phi(r, \xi)|^{2} d \xi \\
\leqslant d \sup _{\eta} \int_{0}^{1} M_{l, d-1}|\Phi|^{2} d \xi+\frac{\varepsilon}{2} \int_{0}^{1} \int_{r}^{\sigma}\left|\frac{\partial \Phi}{\partial \xi}\right|^{2} d \eta d \xi \\
+\kappa \sum_{m^{\prime}=0}^{m}\left(\varepsilon^{-1} \sigma\right)^{m-m^{\prime}}\left(C\left(m^{\prime}\right)\right)^{2} \\
+\left[\sup _{\eta} \int_{0}^{1} M_{l, d}|\Phi(\eta, \xi)|^{2} d \xi\right]^{1 / 2} \int_{r}^{\sigma}\left[\int_{0}^{1} M_{l, d}|H|^{2} d \xi\right]^{1 / 2} d \eta .
\end{aligned}
$$

Беря верхнюю грань по $r$, аналогично находим

$$
\begin{gathered}
\varepsilon \int_{0}^{1} \int_{r}^{\sigma} M_{l, d}\left|\frac{\partial \Phi}{\partial \xi}\right|^{2} d \eta d \xi+d \int_{0}^{1} \int_{r}^{\sigma} M_{l, d-1}\left(\frac{\sigma-\eta}{\sigma^{2}}\right)|\Phi|^{2} d \eta d \xi \\
+\sup _{\eta} \int_{0}^{1} M_{l, d}|\Phi(\eta, \xi)|^{2} d \xi
\end{gathered}
$$




$$
\begin{aligned}
& \leqslant \kappa d \sup _{\eta} \int_{0}^{1} M_{l, d-1}|\Phi|^{2} d \xi+\kappa \varepsilon \int_{0}^{1} \int_{r}^{\sigma}\left|\frac{\partial \Phi}{\partial \xi}\right|^{2} d \eta d \xi \\
& +\kappa \sum_{m^{\prime}=0}^{m}\left(\varepsilon^{-1} \sigma\right)^{m-m^{\prime}}\left(C\left(m^{\prime}\right)\right)^{2} .
\end{aligned}
$$

Здесь, снова благодаря предположению индукции, члены $d \sup _{\eta} \int_{0}^{1} M_{l, d-1}|\Phi|^{2} d \xi$ и $\varepsilon \int_{0}^{1} \int_{r}^{\sigma}\left|\frac{\partial \Phi}{\partial \xi}\right|^{2} d \eta d \xi$ поглощаются последним членом в правой части (124). Это доказывает (99) для всех $m, l, d \geqslant 0$, и лемма 8 , таким образом, оказывается справедливой.

ЗАмЕчАниЕ 3. Для $C\left(m^{\prime}\right)$, определенного формулой $(97)$ с $h=g$, используя леммы 8, 6 и свойство (46), находим, что

$$
\begin{aligned}
\left(C\left(m^{\prime}\right)\right)^{2} & =\left(\sum_{s=0}^{m^{\prime}} \int_{-\infty}^{\sigma}\left[\int_{0}^{1} \mathscr{M}_{l, d}\left|\frac{\partial^{s} g}{\partial \eta^{s}}\right|^{2} d \xi\right]^{1 / 2} d \eta\right)^{2} \\
& \leqslant \kappa\left(\sigma^{2 p+q-m^{\prime}}\right)^{2}\left(\int_{-\sigma}^{\sigma}\left[\int_{0}^{\widetilde{\sigma}} d \xi\right]^{1 / 2} d \eta\right)^{2} \\
& \leqslant \kappa\left(\sigma^{2 p+q+2-m^{\prime}}\right)^{2} .
\end{aligned}
$$

Лемма 9. Предположим, что выполняется (93), т.е. $0<\varepsilon \sigma^{-3} \leqslant 1$. Пусть

$$
\Phi^{m, i}=\frac{\partial^{m} \varphi^{2 p+q+2 i}}{\partial \eta^{m}},
$$

где

$$
u^{\varepsilon} \sim \sum_{i=0}^{\infty} \sigma^{2 i} \varphi^{2 p+q+2 i}
$$

и $\varphi^{j}$ такие, как в (84)-(86) (напомним, что $\varphi^{j}=0$, если $j<2 p+q$ или $j-2 p-q$ нечетно - см. замечание 2). Тогда существует постоянная $\kappa>0$, не зависящая от $\sigma, \varepsilon$, такая, что

$$
\begin{gathered}
\varepsilon \int_{D^{\prime}} M_{l, d}\left|\frac{\partial \Phi^{m, i}}{\partial \xi}\right|^{2} d \eta d \xi+d \sigma^{-1} \int_{D^{\prime}} M_{l, d-1}\left|\Phi^{m, i}\right|^{2} d \eta d \xi \\
\leqslant \kappa \sigma^{i}\left(\varepsilon^{-1} \sigma\right)^{m+i}\left(\sigma^{2 p+q+2}\right)^{2} .
\end{gathered}
$$

ДокАЗАТЕЛЬСтво. Мы будем доказывать (128) индукцией по $i$ и для всех $m$ сразу. Для $i=0$, т. е. $\Phi=\Phi^{m, 0}$ с $m \geqslant 0$, мы отправляемся от (84)-(86) с $j=$ $2 p+q$. Так как $0<\varepsilon \sigma^{-3} \leqslant 1$, то оценка (128) для $i=0$ и $m \geqslant 0$ следует из (96) и замечания 3 .

Предположим, что (128) выполняется при всех $m \geqslant 0$ для порядков, меньших или равных $i-1$. Требуется доказать это для порядка $i$ при всех $m$. Из (84)-(86) с $j=2 p+q+2 i, i \geqslant 1$, записывая (97)-(99) для $\varphi=\varphi^{j}=\varphi^{2 p+q+2 i}$ 
с заменой $h$ на $R_{\varphi}^{j}$, и из (96) аналогично находим, что

$$
\begin{gathered}
\varepsilon \int_{D^{\prime}} M_{l, d}\left|\frac{\partial \Phi^{m, i}}{\partial \xi}\right|^{2} d \eta d \xi+d \sigma^{-1} \int_{D^{\prime}} M_{l, d-1}\left|\Phi^{m, i}\right|^{2} d \eta d \xi \\
\leqslant \kappa \sum_{m^{\prime}=0}^{m}\left(\varepsilon^{-1} \sigma\right)^{m-m^{\prime}} \sum_{s=0}^{m^{\prime}} \int_{D^{\prime}} \mathscr{M}_{l, d}\left|H^{s}\right|^{2} d \eta d \xi
\end{gathered}
$$

где

$$
\begin{aligned}
H^{s}=\varepsilon \sigma^{-2} & \sum_{k=0}^{i-1}(k+1)\left(\frac{\xi}{\sigma^{2}}\right)^{k} \Phi^{s+2, i-k-1}-\varepsilon \sigma^{-2} \sum_{k=0}^{i-1}\left(\frac{\xi}{\sigma^{2}}\right)^{k} \frac{\partial \Phi^{s, i-k-1}}{\partial \xi} \\
& -\sigma^{-2} \sum_{r=0}^{s}\left(\begin{array}{l}
s \\
r
\end{array}\right) \frac{\partial^{s-r}}{\partial \eta^{s-r}}(\sin \eta-\eta) \frac{\partial \Phi^{r, i-1}}{\partial \xi}+\sum_{k=1}^{i}\left(\frac{\xi}{\sigma^{2}}\right)^{k} \Phi^{s+1, i-k} \\
& +\sigma^{-2} \sum_{k=0}^{i-1}\left(\frac{\xi}{\sigma^{2}}\right)^{k} \sum_{r=0}^{s}\left(\begin{array}{l}
s \\
r
\end{array}\right) \frac{\partial^{s-r}}{\partial \eta^{s-r}}\left((1-\cos \eta) \Phi^{r+1, i-k-1}\right)
\end{aligned}
$$

Следовательно, записывая

$$
\begin{aligned}
E_{l, d}\left(\Phi^{m, i}\right)= & \sum_{m^{\prime}=0}^{m} \sum_{i^{\prime}=0}^{i}\left\{\varepsilon \int_{D^{\prime}} \mathscr{M}_{l, d}\left|\frac{\partial \Phi^{m^{\prime}, i^{\prime}}}{\partial \xi}\right|^{2} d \eta d \xi\right. \\
& \left.+d \sigma^{-1} \int_{D^{\prime}} \mathscr{M}_{l, d-1}\left|\Phi^{m^{\prime}, i^{\prime}}\right|^{2} d \eta d \xi\right\}
\end{aligned}
$$

где $l, d \geqslant 0$ - произвольные целые, и используя лемму 7, из (129) и (130) заключаем, что

$$
\begin{aligned}
\int_{D^{\prime}} & \mathscr{M}_{l, d}\left|\varepsilon \sigma^{-2} \sum_{k=0}^{i-1}(k+1)\left(\frac{\xi}{\sigma^{2}}\right)^{k} \Phi^{s+2, i-k-1}\right|^{2} d \eta d \xi \\
& \stackrel{(*)}{\leqslant} \kappa\left(\varepsilon \sigma^{-2}\right)^{2} \sigma \sigma^{-1} \int_{D^{\prime}} \mathscr{M}_{l, d+2(i-1)} \sum_{k=0}^{i-1}\left|\Phi^{s+2, i-k-1}\right|^{2} d \eta d \xi \\
\leqslant & \kappa\left(\varepsilon \sigma^{-2}\right)^{2} \sigma E_{l, d+2 i-1}\left(\Phi^{s+2, i-1}\right)
\end{aligned}
$$

(в $\stackrel{(*)}{\leqslant}$ мы воспользовались тем, что $\mathscr{M}_{l_{1}, d_{1}} \leqslant \kappa \mathscr{M}_{l_{2}, d_{2}}$ для $\left.l_{1} \leqslant l_{2}, d_{1} \leqslant d_{2}\right)$. Второй и четвертый члены в правой части (130) можно оценить аналогично. Для оценки третьего и пятого членов необходимы следующие неравенства:

$$
\begin{aligned}
& \left|\frac{\partial^{s-r}}{\partial \eta^{s-r}}(\sin \eta-\eta)\right| \leqslant \kappa \min \left\{1,(\sigma-\eta)^{3-s+r}+\sigma^{3-s+r}\right\}, \\
& \left|\frac{\partial^{s-r}}{\partial \eta^{s-r}}(1-\cos \eta)\right| \leqslant \kappa \min \left\{1,(\sigma-\eta)^{2-s+r}+\sigma^{2-s+r}\right\} .
\end{aligned}
$$


Чтобы получить, например, оценку (133), используем теорему о разложении Тейлора, гарантирующую существование точки $\alpha$ между 0 и $\eta$ такой, что

$$
f^{(m)}(\eta)=\sum_{k=0}^{n-1} \frac{f^{(m+k)}(0)}{k !} \eta^{k}+\frac{f^{(m+n)}(\alpha)}{n !} \eta^{n} .
$$

Тогда для $f(\eta)=\sin \eta-\eta$ легко увидеть сначала, что $\left|\frac{\partial^{m} f(\eta)}{\partial \eta^{m}}\right| \leqslant \kappa$. Так как $f^{(m+k)}(0)=0$ для $0 \leqslant m+k \leqslant 2$, также заметим, что для $n=3-m \geqslant 0$

$$
\left|\frac{\partial^{m} f(\eta)}{\partial \eta^{m}}\right|=\left|\frac{f^{(3)}(\alpha)}{(3-m) !} \eta^{3-m}\right| \leqslant \kappa|\eta|^{3-m} .
$$

Затем находим, что $\left|\frac{\partial^{s-r} f(\eta)}{\partial \eta^{s-r}}\right| \leqslant \kappa \min \left\{1,|\eta|^{3-s+r}\right\}$, и это влечет за собой (133). Аналогично оцениваем (134).

Отсюда получаем, что

$$
\begin{aligned}
\int_{D^{\prime}} \mathscr{M}_{l, d}\left|\sigma^{-2} \sum_{r=0}^{s}\left(\begin{array}{c}
s \\
r
\end{array}\right) \frac{\partial^{s-r}}{\partial \eta^{s-r}}(\sin \eta-\eta) \frac{\partial \Phi^{r, i-1}}{\partial \xi}\right|^{2} d \eta d \xi \\
\leqslant \kappa\left(\sigma^{-2}\right)^{2} \varepsilon^{-1} \varepsilon\left\{\int_{D^{\prime}} \mathscr{M}_{l, d} \sum_{r=0}^{s-3}\left|\frac{\partial \Phi^{r, i-1}}{\partial \xi}\right|^{2} d \eta d \xi\right. \\
\left.\quad+\int_{D^{\prime}} \sum_{r=s-2}^{s} \sigma^{2(3-s+r)}\left(\mathscr{M}_{l+2(3-s+r), d}+\mathscr{M}_{l, d}\right)\left|\frac{\partial \Phi^{r, i-1}}{\partial \xi}\right|^{2} d \eta d \xi\right\} \\
\leqslant \kappa \sigma^{-4} \varepsilon^{-1}\left(E_{l, d+1}\left(\Phi^{s-3, i-1}\right)+\sum_{r=s-2}^{s} \sigma^{2(3-s+r)} E_{l+6, d+1}\left(\Phi^{r, i-1}\right)\right) .
\end{aligned}
$$

Аналогично оценивается пятый член в правой части (130). Таким образом, можно вывести следующие оценки:

$$
\begin{aligned}
\int_{D^{\prime}} \mathscr{M}_{l, d} \mid & \left.H^{s}\right|^{2} d \eta d \xi \\
\leqslant & \kappa\left(\left(\varepsilon \sigma^{-2}\right)^{2} \sigma E_{l, d+2 i-1}\left(\Phi^{s+2, i-1}\right)+\left(\varepsilon \sigma^{-2}\right)^{2} \varepsilon^{-1} E_{l, d+2 i-1}\left(\Phi^{s, i-1}\right)\right. \\
& +\sigma^{-4} \varepsilon^{-1}\left(E_{l, d+1}\left(\Phi^{s-3, i-1}\right)+\sum_{r=s-2}^{s} \sigma^{2(3-s+r)} E_{l+6, d+1}\left(\Phi^{r, i-1}\right)\right) \\
& +\sigma E_{l, d+2 i+1}\left(\Phi^{s+1, i-1}\right) \\
& \left.+\sigma^{-4} \sigma\left(E_{l, d+2 i-1}\left(\Phi^{s-1, i-1}\right)+\sum_{r=s-1}^{s} \sigma^{2(2-s+r)} E_{l+4, d+2 i-1}\left(\Phi^{r+1, i-1}\right)\right)\right)
\end{aligned}
$$

где $E_{l, d}$ определено формулой (131). По предположению индукции для (128) заметим, что для порядков $\leqslant i-1$

$$
E_{l, d}\left(\Phi^{m, i-1}\right) \leqslant \kappa\left(\varepsilon^{-1} \sigma\right)^{m+i-1} \sigma^{i-1}\left(\sigma^{2 p+q+2}\right)^{2} \quad \text { для всех } \quad l, d \geqslant 0 .
$$


Из (137), используя $0<\varepsilon \sigma^{-3} \leqslant 1$, получим, что для $j=2 p+q+2 i,(\eta, \xi) \in$ $(-\infty, \sigma) \times(0,1)$

$$
\begin{gathered}
\int_{D^{\prime}} \mathscr{M}_{l, d}\left|H^{s}\right|^{2} d \eta d \xi \leqslant \kappa\left(\varepsilon \sigma^{-3}+\varepsilon^{2} \sigma^{-6}+\sigma^{-5} \varepsilon^{-1}\left(\varepsilon^{4} \sigma^{-4}+\varepsilon \sigma^{5} \sum_{r=s-2}^{s}\left(\varepsilon \sigma^{-3}\right)^{s-r}\right)\right. \\
\left.+1+\sigma^{-4}\left(\varepsilon^{2} \sigma^{-2}+\sigma^{4} \sum_{r=s-1}^{s}\left(\varepsilon \sigma^{-3}\right)^{s-r}\right)\right)\left(\varepsilon^{-1} \sigma\right)^{s+i} \sigma^{i}\left(\sigma^{2 p+q+2}\right)^{2} \\
\leqslant \kappa\left(\varepsilon^{-1} \sigma\right)^{s+i} \sigma^{i}\left(\sigma^{2 p+q+2}\right)^{2} .
\end{gathered}
$$

Следовательно, правая часть (129) ограничена величиной

$$
\kappa \sum_{m^{\prime}=0}^{m}\left(\varepsilon^{-1} \sigma\right)^{m-m^{\prime}} \sum_{s=0}^{m^{\prime}}\left(\varepsilon^{-1} \sigma\right)^{s+i} \sigma^{i}\left(\sigma^{2 p+q+2}\right)^{2} \leqslant \kappa\left(\varepsilon^{-1} \sigma\right)^{m+i} \sigma^{i}\left(\sigma^{2 p+q+2}\right)^{2} .
$$

Это доказывает оценку (128) для любого порядка $i$ с $m \geqslant 0$. Лемма 9 доказана.

Таким образом, построены параболические слои, производимые задачей (66), соответствующие $\widehat{f}-f^{*}$, т. е. задачей

$$
\left\{\begin{array}{l}
L_{\varepsilon} u^{\varepsilon}=\widehat{f}-f^{*}=(1-\rho(x) \check{\rho}(x))(1+x) C_{u}(x)^{2 p} y^{q} \quad \text { в } D, \\
u^{\varepsilon}=0 \text { на } \partial D
\end{array}\right.
$$

и для них получены оценки.

Изучив параболические пограничные слои, возникающие в точке $\eta=0$, $\xi=0$, используем их для того, чтобы получить оценку решения $u^{\varepsilon}$ задачи $(67)$ (и, стало быть, (66)), которая обсуждается в следующем разделе.

\section{5. Анализ сходимости для задачи (66)}

Напомним, что $\varphi^{j}=0$, если $j<2 p+q$ или $j=2 p+q+2 i+1, i \geqslant 0$, а в противном случае $\varphi^{j}$ рекуррентно определено в (84)-(86). После изучения параболических пограничных слоев, образованных в $\eta=0, \xi=0$, используем их для того, чтобы получить оценку решения $u^{\varepsilon}$ задачи (67). Записывая

$$
\varphi_{\varepsilon n}=\sum_{i=0}^{n} \sigma^{2 i} \varphi^{, 2 i}, \quad \varphi^{, 2 i}=\varphi^{2 p+q+2 i},
$$

умножая (84) на $\sigma^{2 i}$ и суммируя получившиеся уравнения от $i=0$ до $n$, находим, что

$$
\begin{aligned}
& -\varepsilon \frac{\partial^{2} \varphi_{\varepsilon n}}{\partial \xi^{2}}+\eta \frac{\partial \varphi_{\varepsilon n}}{\partial \xi}-\frac{\partial \varphi_{\varepsilon n}}{\partial \eta}=g(\eta, \xi) \\
& \quad+\varepsilon \sum_{i=0}^{n} \sum_{k=0}^{i-1}(k+1) \xi^{k} \sigma^{2 i-2 k-2} \frac{\partial^{2} \varphi^{, 2 i-2 k-2}}{\partial \eta^{2}} \\
& \quad-\varepsilon \sum_{i=0}^{n} \sum_{k=0}^{i-1} \xi^{k} \sigma^{2 i-2 k-2} \frac{\partial \varphi^{, 2 i-2 k-2}}{\partial \xi}-(\sin \eta-\eta) \sum_{i=0}^{n} \sigma^{2 i-2} \frac{\partial \varphi^{, 2 i-2}}{\partial \xi} \\
& \quad+\sum_{i=0}^{n} \sum_{k=1}^{i} \xi^{k} \sigma^{2 i-2 k} \frac{\partial \varphi^{, 2 i-2 k}}{\partial \eta}-(1-\cos \eta) \sum_{i=0}^{n} \sum_{k=0}^{i-1} \xi^{k} \sigma^{2 i-2 k-2} \frac{\partial \varphi^{, 2 i-2 k-2}}{\partial \eta}
\end{aligned}
$$


Переставляя суммы по $k$ и $i$, получим:

$$
\begin{aligned}
& -\varepsilon \frac{\partial^{2} \varphi_{\varepsilon n}}{\partial \xi^{2}}+\eta \frac{\partial \varphi_{\varepsilon n}}{\partial \xi}-\frac{\partial \varphi_{\varepsilon n}}{\partial \eta}=g(\eta, \xi)+\varepsilon \sum_{m=0}^{n-1} \sigma^{2 m} \frac{\partial^{2} \varphi^{2 m}}{\partial \eta^{2}} \sum_{k=0}^{n-1-m}(k+1) \xi^{k} \\
& -\varepsilon \sum_{m=0}^{n-1} \sigma^{2 m} \frac{\partial \varphi^{, 2 m}}{\partial \xi} \sum_{k=0}^{n-1-m} \xi^{k}-(\sin \eta-\eta) \sum_{m=0}^{n-1} \sigma^{2 m} \frac{\partial \varphi^{, 2 m}}{\partial \xi} \\
& \quad+\sum_{m=0}^{n} \sigma^{2 m} \frac{\partial \varphi^{, 2 m}}{\partial \eta} \sum_{k=0}^{n-m} \xi^{k}-\frac{\partial \varphi_{\varepsilon n}}{\partial \eta}-(1-\cos \eta) \sum_{m=0}^{n-1} \sigma^{2 m} \frac{\partial \varphi^{, 2 m}}{\partial \eta} \sum_{k=0}^{n-1-m} \xi^{k},
\end{aligned}
$$

что влечет

$$
\begin{aligned}
A:= & -\varepsilon \frac{\partial^{2} \varphi_{\varepsilon n}}{\partial \xi^{2}}+\eta \frac{\partial \varphi_{\varepsilon n}}{\partial \xi} \\
= & g(\eta, \xi)+\varepsilon \frac{\partial^{2} \varphi_{\varepsilon, n-1}}{\partial \eta^{2}} \sum_{k=0}^{n-1-m}(k+1) \xi^{k}-\varepsilon \frac{\partial \varphi_{\varepsilon, n-1}}{\partial \xi} \sum_{k=0}^{n-1-m} \xi^{k} \\
& -(\sin \eta-\eta) \frac{\partial \varphi_{\varepsilon, n-1}}{\partial \xi}+\sigma^{2 n} \frac{\partial \varphi^{, 2 n}}{\partial \eta}+\cos \eta \frac{\partial \varphi_{\varepsilon, n-1}}{\partial \eta} \sum_{k=0}^{n-1-m} \xi^{k}
\end{aligned}
$$

Отсюда находим, что

$$
\begin{aligned}
L_{\varepsilon} \varphi_{\varepsilon n} & =-\frac{\varepsilon}{(1-\xi)^{2}} \frac{\partial^{2} \varphi_{\varepsilon n}}{\partial \eta^{2}}+\frac{\varepsilon}{1-\xi} \frac{\partial \varphi_{\varepsilon n}}{\partial \xi}+A+(\sin \eta-\eta) \frac{\partial \varphi_{\varepsilon n}}{\partial \xi}-\frac{\cos \eta}{1-\xi} \frac{\partial \varphi_{\varepsilon n}}{\partial \eta} \\
& =g(\eta, \xi)+\operatorname{Rem},
\end{aligned}
$$

где

$$
\begin{aligned}
\operatorname{Rem}=- & \frac{\varepsilon}{(1-\xi)^{2}} \sigma^{2 n} \frac{\partial^{2} \varphi^{, 2 n}}{\partial \eta^{2}}-\varepsilon \sum_{m=0}^{n-1} \sigma^{2 m} \frac{\partial^{2} \varphi^{, 2 m}}{\partial \eta^{2}} \sum_{k=n-m}^{\infty}(k+1) \xi^{k} \\
& +\frac{\varepsilon}{1-\xi} \sigma^{2 n} \frac{\partial \varphi^{, 2 n}}{\partial \xi}+\varepsilon \sum_{m=0}^{n-1} \sigma^{2 m} \frac{\partial \varphi^{, 2 m}}{\partial \xi} \sum_{k=n-m}^{\infty} \xi^{k} \\
& +(\sin \eta-\eta) \sigma^{2 n} \frac{\partial \varphi^{, 2 n}}{\partial \xi}+\sigma^{2 n} \frac{\partial \varphi^{, 2 n}}{\partial \eta}+\cos \eta \sigma^{2 n} \frac{\partial \varphi^{, 2 n}}{\partial \eta} \\
& -\cos \eta \sum_{m=0}^{n-1} \sigma^{2 m} \frac{\partial \varphi^{, 2 m}}{\partial \eta} \sum_{k=n-m}^{\infty} \xi^{k} .
\end{aligned}
$$

Для бесконечных рядов в (147) используем следующие обстоятельства:

$$
\begin{aligned}
\sum_{k=n-m}^{\infty}(k+1) \xi^{k} & =\xi^{n-m}\left(\sum_{k=0}^{\infty}(k+1) \xi^{k}+(n-m) \sum_{k=0}^{\infty} \xi^{k}\right) \\
& =\xi^{n-m}\left((1-\xi)^{-2}+(n-m)(1-\xi)^{-1}\right) \\
\sum_{k=n-m}^{\infty} \xi^{k} & =\xi^{n-m}(1-\xi)^{-1} .
\end{aligned}
$$


Поскольку пограничные слои появляются локально около границ, то, чтобы понять их поведение вдали от границ, мы используем приближенную форму $\bar{\varphi}, 2 i$ для $\varphi^{2 i}$. Определим

$$
\bar{\varphi}_{\varepsilon n}=\sum_{i=0}^{n} \sigma^{2 i} \bar{\varphi}^{, 2 i}, \quad \bar{\varphi}^{, 2 i}=\varphi^{2 p+q+2 i} \delta(\xi) \varrho(\eta),
$$

где $\delta=\delta(\xi)$ и $\varrho=\varrho(\eta)$ (не путать с $\rho$ в (43)!) - гладкие обрезывающие функции, $0 \leqslant \delta, \varrho \leqslant 1$, такие, что $\delta=0$, если $\xi \geqslant 1 / 2, \delta=1$, если $\xi \leqslant 1 / 4, \varrho=0$, если $\eta \leqslant-\pi / 2$, и $\varrho=1$, если $\eta \geqslant-\pi / 4$.

Получим оценки для $\varphi^{2 i}$ в разных нормах. Напомним, что $\varphi^{2 i}=\varphi^{2 p+q+2 i}-$ решения уравнения (84).

Лемма 10. Предположим, что выполнено (93). Пусть $\varphi^{2 i}=\varphi^{2 p+q+2 i}$ решения уравнения (84) с (85) и (86). При $j=2 p+q+2 i, i \geqslant 0$, существует постоянная $\kappa>0$, не зависящая от $\sigma$ и $\varepsilon$, такая, что

$$
\begin{aligned}
& \varepsilon^{1 / 2}\left|\eta^{r} \xi^{s} \frac{\partial}{\partial \xi}\left[\frac{\partial^{m} \varphi^{, 2 i}}{\partial \eta^{m}}\right]\right|_{L^{2}\left(D^{\prime}\right)}+\sigma^{-1 / 2}\left|\eta^{r} \xi^{s} \frac{\partial^{m} \varphi^{, 2 i}}{\partial \eta^{m}}\right|_{L^{2}\left(D^{\prime}\right)} \\
& \leqslant \kappa\left(\varepsilon^{-1} \sigma\right)^{(m+i) / 2} \sigma^{r+2 s+2 p+q+2+i / 2} \quad \partial \Omega я \quad r, s \geqslant 0, \\
& \varepsilon^{1 / 2}\left|\frac{\partial}{\partial \xi}\left[\frac{\partial^{m}\left(\bar{\varphi}^{, 2 i}-\varphi^{, 2 i}\right)}{\partial \eta^{m}}\right]\right|_{L^{2}\left(D^{\prime}\right)}+\sigma^{-1 / 2}\left|\frac{\partial^{m}\left(\bar{\varphi}^{, 2 i}-\varphi^{, 2 i}\right)}{\partial \eta^{m}}\right|_{L^{2}\left(D^{\prime}\right)} \\
& \leqslant \kappa\left(\varepsilon^{-1} \sigma\right)^{(m+i) / 2} \sigma^{2 s+2 p+q+2+i / 2} \quad \text { для произвольных } s \geqslant 0 .
\end{aligned}
$$

Здесъ, напомним, $D^{\prime}=(-\infty, \sigma) \times(0,1)$.

ДоказАтельство. Заметим, что

$$
\begin{aligned}
\sigma^{-1}\left|\eta^{r} \xi^{s} \frac{\partial^{m} \varphi^{, 2 i}}{\partial \eta^{m}}\right|_{L^{2}\left(D^{\prime}\right)}^{2} & \left.\leqslant \kappa \sigma^{-1} \int_{D^{\prime}}\left(\sigma^{2 r}+(\sigma-\eta)\right)^{2 r}\right) \xi^{2 s}\left|\frac{\partial^{m} \varphi^{, 2 i}}{\partial \eta^{m}}\right|^{2} \\
& \leqslant \kappa \sigma^{-1} \int_{D^{\prime}} \sigma^{2 r+4 s}\left(M_{0,2 s}+M_{2 r, 2 s}\right)\left|\frac{\partial^{m} \varphi^{, 2 i}}{\partial \eta^{m}}\right|^{2} .
\end{aligned}
$$

Из леммы 9 получим нужную границу для второго члена в левой части неравенства (150). Первый член в левой части (150) оценивается аналогично. Чтобы доказать (151), заметим, что

$$
\begin{aligned}
\sigma^{-1 / 2} & \left|\frac{\partial^{m}\left(\bar{\varphi}^{, 2 i}-\varphi^{, 2 i}\right)}{\partial \eta^{m}}\right|_{L^{2}\left(D^{\prime}\right)} \leqslant \kappa \sigma^{-1 / 2} \sum_{k=0}^{m}\left|\left(M_{r, 0}+M_{0, s}\right)\right| \frac{\partial^{k} \varphi^{, 2 i}}{\partial \eta^{k}}||_{L^{2}\left(D^{\prime}\right)} \\
& \times\left|\left(M_{r, 0}+M_{0, s}\right)^{-1}\right|_{L^{\infty}\left(D^{\prime} \backslash(-\pi / 4, \sigma) \times(0,1 / 4)\right)} .
\end{aligned}
$$

Полагая $r=2 s$ в (153) и используя лемму 9, получим требуемую оценку для второго члена в левой части (151); первый член оценивается аналогично. Лемма 10 доказана.

Запишем теперь $w_{0}=u^{\varepsilon}-\bar{\varphi}_{\varepsilon n}$, где $u^{\varepsilon}-$ решение задачи (67), и возьмем скалярное произведение $L_{\varepsilon}\left(w_{0}\right)$ с $e^{y} w_{0}$. Чтобы справиться с сингулярными множителями $(1-\xi)^{-1},(1-\xi)^{-2}$ в члене Rem в (147)-(148), введем гладкую функцию $\widetilde{\delta}=1-\delta(1-\xi)$ такую, что $\widetilde{\delta}=1$, если $\xi \leqslant 1 / 2$, и $\widetilde{\delta}=0$, если $\xi \geqslant 3 / 4$. 
Заметим затем, что $L_{\varepsilon}\left(w_{0}\right)=g(\eta, \xi)-L_{\varepsilon}\left(\bar{\varphi}_{\varepsilon n}\right)$ имеет компактный носитель в области $\xi \leqslant 1 / 2$, и, следовательно,

$$
\begin{aligned}
\left|\left(L_{\varepsilon}\left(w_{0}\right), e^{y} w_{0}\right)\right| & =\left|\left(L_{\varepsilon}\left(w_{0}\right), e^{y} w_{0} \widetilde{\delta}\right)\right| \\
& =\left|\left(L_{\varepsilon}\left(u^{\varepsilon}-\varphi_{\varepsilon n}\right), e^{y} w_{0} \widetilde{\delta}\right)-\left(L_{\varepsilon}\left(\bar{\varphi}_{\varepsilon n}-\varphi_{\varepsilon n}\right), e^{y} w_{0} \widetilde{\delta}\right)\right| \\
& \leqslant\left|\left(\operatorname{Rem}, e^{y} w_{0} \widetilde{\delta}\right)\right|+\left|\left(L_{\varepsilon}\left(\bar{\varphi}_{\varepsilon n}-\varphi_{\varepsilon n}\right), e^{y} w_{0} \widetilde{\delta}\right)\right| .
\end{aligned}
$$

Из леммы 10 получим, что

$$
\begin{aligned}
&\left|\left(\operatorname{Rem}, e^{y} w_{0} \widetilde{\delta}\right)\right|=\left|\int_{D} \operatorname{Rem} e^{y} w_{0} \widetilde{\delta}\right| \\
& \leqslant \kappa\left\{\varepsilon \sigma^{2 n}\left|\frac{\partial^{2} \varphi^{, 2 n}}{\partial \eta^{2}}\right|_{L^{2}\left(D^{\prime}\right)}+\varepsilon\left|\sum_{m=0}^{n-1} \sigma^{2 m} \xi^{n-m} \frac{\partial^{2} \varphi^{, 2 m}}{\partial \eta^{2}}\right|_{L^{2}\left(D^{\prime}\right)}\right. \\
&+\varepsilon \sigma^{2 n}\left|\frac{\partial \varphi^{, 2 n}}{\partial \xi}\right|_{L^{2}\left(D^{\prime}\right)}+\varepsilon\left|\sum_{m=0}^{n-1} \sigma^{2 m} \xi^{n-m} \frac{\partial \varphi^{, 2 m}}{\partial \xi}\right|_{L^{2}\left(D^{\prime}\right)} \\
&+\sigma^{2 n}\left|\eta^{3} \frac{\partial \varphi^{, 2 n}}{\partial \xi}\right|_{L^{2}\left(D^{\prime}\right)}+\sigma^{2 n}\left|\frac{\partial \varphi^{, 2 n}}{\partial \eta}\right|_{L^{2}\left(D^{\prime}\right)} \\
&\left.+\left|\sum_{m=0}^{n-1} \sigma^{2 m} \xi^{n-m} \frac{\partial \varphi^{, 2 m}}{\partial \eta}\right|_{L^{2}\left(D^{\prime}\right)}\right\}\left|w_{0}\right|_{L^{2}(D)} \\
& \leqslant \kappa\left(\varepsilon^{-1} \sigma^{6}\right)^{n / 2} \sigma^{2 p+q+2} \\
& \times\left\{\sigma^{3 / 2}+\varepsilon^{1 / 2} \sigma^{1 / 2}+\varepsilon^{1 / 2}+\varepsilon \sigma^{-1}+\varepsilon^{-1 / 2} \sigma^{3}+\varepsilon^{-1 / 2} \sigma+1\right\}\left|w_{0}\right|_{L^{2}(D)} .
\end{aligned}
$$

Так как $0<\varepsilon \sigma^{-3} \leqslant 1$, находим, что

$$
\left|\left(\operatorname{Rem}, e^{y} w_{0} \widetilde{\delta}\right)\right| \leqslant \kappa\left(\varepsilon^{-1} \sigma^{6}\right)^{n / 2} \varepsilon^{-1 / 2} \sigma^{2 p+q+3}\left|w_{0}\right|_{L^{2}(D)} .
$$

Ввиду (151) имеем

$$
\begin{aligned}
& \left|\left(L_{\varepsilon}\left(\bar{\varphi}_{\varepsilon n}-\varphi_{\varepsilon n}\right), e^{y} w_{0} \widetilde{\delta}\right)\right| \\
& \quad \leqslant\left|\nabla_{\eta, \xi}\left(\bar{\varphi}_{\varepsilon n}-\varphi_{\varepsilon n}\right)\right|_{L^{2}\left(D^{\prime}\right)}\left(\varepsilon\left|w_{0}\right|_{H^{1}(D)}+\left|w_{0}\right|_{L^{2}(D)}\right) \\
& \quad \leqslant \kappa \varepsilon^{-1 / 2} \sigma^{2 s+2 p+q+2} \sum_{i=0}^{n}\left(\varepsilon^{-1} \sigma^{6}\right)^{i / 2}\left(\varepsilon\left|w_{0}\right|_{H^{1}(D)}+\left|w_{0}\right|_{L^{2}(D)}\right) .
\end{aligned}
$$

Выберем $\sigma$ такое, что кроме $(93)$ выполнено неравенство $\varepsilon^{-1} \sigma^{6} \leqslant 1$ и, таким образом,

$$
\sigma^{6} \leqslant \varepsilon \leqslant \sigma^{3}, \quad \text { т. е. } \quad \varepsilon^{1 / 3} \leqslant \sigma \leqslant \varepsilon^{1 / 6} .
$$

Тогда

$$
\left|\left(L_{\varepsilon}\left(\bar{\varphi}_{\varepsilon n}-\varphi_{\varepsilon n}\right), e^{y} w_{0} \widetilde{\delta}\right)\right| \leqslant \kappa \varepsilon^{-1 / 2} \sigma^{2 s+2 p+q+2}\left(\varepsilon\left|w_{0}\right|_{H^{1}(D)}+\left|w_{0}\right|_{L^{2}(D)}\right) .
$$

Здесь $s \geqslant 0$ произвольно. Поэтому член (159) может иметь произвольно малый порядок. Из (154) получим следующую теорему.

Теорема 6. Пусть и $u^{\varepsilon}$ - решения задачи (67), и пусть $\bar{\varphi}_{\varepsilon п}$ определено формулой (149). Предположим, что выполнено условие (158). Тогда существует постоянная $\kappa>0$, не зависящая от $\sigma, \varepsilon$, такая, что

$$
\left\|u^{\varepsilon}-\bar{\varphi}_{\varepsilon n}\right\|_{\varepsilon} \leqslant \kappa\left(\varepsilon^{-1} \sigma^{6}\right)^{n / 2} \varepsilon^{-1 / 2} \sigma^{2 p+q+3} .
$$


Комбинируя этот результат с теоремой 5 , получим следующую теорему о сходимости для уравнения (40). Прежде чем ее сформулировать, напомним следующие обозначения.

(A) $u_{\varepsilon n}^{*}=u_{\varepsilon n}^{*}[p, q]=\sum_{j=0}^{n} \varepsilon^{j} u^{* j}$, где

$$
u^{* j}=\int_{y}^{C_{u}(x)} \Delta u^{*, j-1}(x, s) d s
$$

и $\Delta u^{*,-1}=f^{*}$.

(B) $\bar{\theta}_{\varepsilon n}^{*}=\bar{\theta}_{\varepsilon n}^{*}[p, q]=\sum_{j=0}^{n} \varepsilon^{j} \theta^{* j} \delta(\xi)$, где

$$
\theta^{* j}=P^{j}(\eta, \bar{\xi}) \exp ((\sin \eta) \bar{\xi}) \chi_{[\pi, 2 \pi]}(\eta),
$$

и $P^{j}(\eta, \bar{\xi})$ такие же, как в $(34),(35)$, с заменой $v^{j}(\eta)$ на $v^{* j}(\eta)=-u^{* j}(\cos \eta, \sin \eta)$ и для $2 p+q \leqslant 3 n-1$.

(C1) Для $2 p+q<3 n$

$$
\bar{\varphi}_{\varepsilon n}=\bar{\varphi}_{\varepsilon n}[p, q]=\sum_{i=0}^{n} \sigma^{2 i} \bar{\varphi}^{, 2 i}, \quad \bar{\varphi}^{, 2 i}=\varphi^{2 p+q+2 i} \delta(\xi) \varrho(\eta)
$$

из (149). Здесь пограничные слои $\varphi^{2 p+q+2 i}$, а также $\delta(\xi)$ и $\varrho(\eta)$ относятся к задаче (67). Можно аналогичным образом построить параболические пограничные слои $\varphi^{2 p+q+2 i}$ с $\delta(\xi)$ и $\varrho(\eta)$, отвечающими

$$
f^{* *}=(1-\check{\rho}(x))(1+x) C_{u}(x)^{2 p} y^{q} .
$$

Отсюда, складывая оба параболических пограничных слоя, получим $\bar{\varphi}, 2 i$ соответствующим образом определенным для уравнения (66).

(C2) Для $2 p+q \geqslant 3 n$ положим $\bar{\varphi}_{\varepsilon n}=\bar{\varphi}_{\varepsilon n}[p, q]=0$.

Итак, докажем следующую теорему.

TеOрема 7. Пусть $\sigma=\varepsilon^{1 / 4}$ (чmо соответствует (158)), и пусть $u^{\varepsilon}-$ решение задачи (40). Тогда существует постоянная $\kappa>0$, не зависящая om $\varepsilon$, такая, что

$$
\left\|u^{\varepsilon}-u_{\varepsilon n}^{*}-\bar{\theta}_{\varepsilon n}^{*}-\bar{\varphi}_{\varepsilon n}\right\|_{\varepsilon} \leqslant \kappa \varepsilon^{(n+2 p+q+1) / 4},
$$

где при $2 p+q<3 n$

$$
\bar{\varphi}_{\varepsilon n}=\sum_{i=0}^{n} \sigma^{2 i} \bar{\varphi}^{, 2 i}=\sum_{i=0}^{n} \varepsilon^{i / 2} \bar{\varphi}, 2 i
$$

u $n p u 2 p+q \geqslant 3 n$

$$
\bar{\varphi}_{\varepsilon n}=0 .
$$

ДокАЗАтельство. Сначала рассмотрим случай $2 p+q \leqslant 3 n-1$. Из теоремы 5 , а также из теоремы 6 при $\bar{\varphi}_{\varepsilon n}=\bar{\varphi}_{\varepsilon m}, m \geqslant 0$, полагая $\left(\varepsilon \sigma^{-3}\right)^{n}=$ $\left(\varepsilon^{-1} \sigma^{6}\right)^{m / 2}$, находим, что

$$
\begin{aligned}
\| u^{\varepsilon}- & u_{\varepsilon n}^{*}-\bar{\theta}_{\varepsilon n}^{*}-\bar{\varphi}_{\varepsilon m} \|_{\varepsilon} \leqslant \kappa\left(\varepsilon \sigma^{-3}\right)^{n} \sigma^{2 p+q} \\
& \times\left[\varepsilon^{-1 / 2} \sigma^{3}+\varepsilon\left(\sigma^{-1}+\min \left\{\varepsilon^{-1 / 2}, \sigma^{-2}\right\}\right)\right] .
\end{aligned}
$$


Полагая $\left(\varepsilon \sigma^{-3}\right)^{n}=\left(\varepsilon^{-1} \sigma^{6}\right)^{m / 2}$, имеем

$$
\sigma=\varepsilon^{(2 n+m) /(6(n+m))} \quad \text { и } \quad\left(\varepsilon \sigma^{-3}\right)^{n}=\varepsilon^{m n /(2(n+m))} .
$$

Заметим, что

$$
\frac{1}{6} \leqslant \frac{2 n+m}{6(n+m)} \leqslant \frac{1}{3}
$$

в соответствии с (158). Для нахождения оптимального выбора $m, n$ заметим, что

$$
\frac{m n}{2(n+m)}=\left(2\left(\frac{1}{m}+\frac{1}{n}\right)\right)^{-1} \leqslant\left(\frac{4}{\sqrt{m n}}\right)^{-1},
$$

так как $(a+b) / 2 \geqslant \sqrt{a b}$ для любых $a, b \geqslant 0$ и равенство достигается при $a=b$. Оптимальным, таким образом, будет выбор $n=m$. Для $2 p+q \leqslant 3 n-1$ находим, что

$$
\sigma=\varepsilon^{1 / 4}, \quad\left(\varepsilon \sigma^{-3}\right)^{n}=\varepsilon^{n / 4},
$$

откуда следует утверждение теоремы.

Для $2 p+q \geqslant 3 n$, используя теорему 5 с $\sigma=\varepsilon^{1 / 4}$ как в (165) получим утверждение теоремы. Теорема 7 доказана.

\section{6. Общий случай}

Рассмотрим случай общей функции $f$, как в (1). Записывая разложение

$$
f=f-\widehat{f}+\rho(x) \check{\rho}(x) \widehat{f}+(1-\rho(x) \check{\rho}(x)) \widehat{f},
$$

мы, в силу линейности, можем воспользоваться всеми результатами из предыдущих разделов. Объединяя все эти результаты, мы получим следующие утверждения о сходимости для произвольной $f$. Сначала рассмотрим общий порядок $n \geqslant 0$.

6.1. Порядок $n \geqslant 0$. Начнем с определений.

(A) $\widetilde{u}_{\varepsilon n}=\sum_{j=0}^{n} \varepsilon^{j} \widetilde{u}^{j}$, где

$$
\widetilde{u}^{j}=\int_{y}^{C_{u}(x)} \Delta \widetilde{u}^{j-1}(x, s) d s
$$

и $\Delta \widetilde{u}^{-1}=\widetilde{f}=f-\widehat{f}$. Здесь $\widehat{f}$ - сумма тейлоровских разложений $f$ порядка $2+3 n$ в точках $(1,0)$ и $(-1,0)$, т. е.

$$
\widehat{f}=\sum_{0 \leqslant 2 p+q \leqslant 2+3 n}\left(\alpha_{p, q, L}(1-x) C_{u}(x)^{2 p} y^{q}+\alpha_{p, q, R}(1+x) C_{u}(x)^{2 p} y^{q}\right),
$$

где

$$
\begin{aligned}
\alpha_{p, q, L} & =\frac{\partial^{p+q} f}{\partial x^{p} \partial y^{q}}(-1,0) \frac{1}{2^{p+1} p ! q !}, \\
\alpha_{p, q, R} & =-\frac{\partial^{p+q} f}{\partial x^{p} \partial y^{q}}(1,0) \frac{1}{(-2)^{p+1} p ! q !} .
\end{aligned}
$$


(B) $\tilde{\theta}_{\varepsilon n}=\sum_{j=0}^{n} \varepsilon^{j} \widetilde{\theta}^{j}$, где

$$
\widetilde{\theta}^{j}=P^{j}(\eta, \bar{\xi}) \exp ((\sin \eta) \bar{\xi}) \delta(\xi) \chi_{[\pi, 2 \pi]}(\eta)
$$

a $P^{j}(\eta, \bar{\xi})$ определены в разделе 1 с $v^{j}(\eta)=-\widetilde{u}^{j}(\cos \eta, \sin \eta)$ из (166).

(C) $\bar{\xi}_{\varepsilon n, R}[p, q]=u_{\varepsilon n, R}^{*}[p, q]+\bar{\theta}_{\varepsilon n, R}^{*}[p, q]+\bar{\varphi}_{\varepsilon n, R}[p, q]$, где, напомним,

$$
\begin{gathered}
u_{\varepsilon n, R}^{*}[p, q]=u_{\varepsilon n}^{*}[p, q], \quad \bar{\theta}_{\varepsilon n, R}^{*}[p, q]=\bar{\theta}_{\varepsilon n}^{*}[p, q], \\
\bar{\varphi}_{\varepsilon n, R}[p, q]=\bar{\varphi}_{\varepsilon n}[p, q]
\end{gathered}
$$

с $\sigma=\varepsilon^{1 / 4}$ определены в разделе 5. Это случай уравнения (66).

(D) Заменяя $x$ на $-x$, мы можем определить $u_{\varepsilon n, L}^{*}[p, q], \bar{\theta}_{\varepsilon n, L}^{*}[p, q], \bar{\varphi}_{\varepsilon n, L}[p, q]$ с $\sigma=\varepsilon^{1 / 4}$, отвечающие задаче $(66)$ с заменой $\widehat{f}-f^{*}$ на

$$
(1-\rho(x) \check{\rho}(x))(1-x) C_{u}(x)^{2 p} y^{q} .
$$

Затем определим

$$
\bar{\xi}_{\varepsilon n, L}[p, q]=u_{\varepsilon n, L}^{*}[p, q]+\bar{\theta}_{\varepsilon n, L}^{*}[p, q]+\bar{\varphi}_{\varepsilon n, L}[p, q] .
$$

Теперь сформулируем и докажем основную теорему.

Теорема 8. Пусть $\sigma=\varepsilon^{1 / 4}$, и пусть $u^{\varepsilon}-$ решение задачи (1). Для целого $n \geqslant 0$ существует постоянная $\kappa>0$, не зависящая от $\varepsilon$, такая, что

$$
\begin{gathered}
\| u^{\varepsilon}-\widetilde{u}_{\varepsilon n}-\widetilde{\theta}_{\varepsilon n}-\sum_{0 \leqslant 2 p+q \leqslant 2+3 n}\left(\alpha_{p, q, L} \bar{\xi}_{\varepsilon, 4 n+3, L}[p, q]\right. \\
\left.\quad+\alpha_{p, q, R} \bar{\xi}_{\varepsilon, 4 n+3, R}[p, q]\right) \|_{\varepsilon} \leqslant \kappa \varepsilon^{n+1} .
\end{gathered}
$$

ДоказАтельство. Пусть $U_{R}^{\varepsilon}-$ решение задачи (40) с заменой $\widehat{f}$ на

$$
\widehat{f}=\sum_{0 \leqslant 2 p+q \leqslant 2+3 n} \alpha_{p, q, R}(1+x) C_{u}(x)^{2 p} y^{q} .
$$

Из теоремы 7 заключаем, что существует постоянная $\kappa>0$, не зависящая от $\varepsilon$, такая, что

$$
\begin{aligned}
\| U_{R}^{\varepsilon} & -\sum_{0 \leqslant 2 p+q \leqslant 2+3 n} \alpha_{p, q, R} \bar{\xi}_{\varepsilon m, R}[p, q] \|_{\varepsilon} \\
& =\left\|U_{R}^{\varepsilon}-\sum_{0 \leqslant 2 p+q \leqslant 2+3 n} \alpha_{p, q, R}\left(u_{\varepsilon m, R}^{*}[p, q]+\bar{\theta}_{\varepsilon m, R}^{*}[p, q]+\bar{\varphi}_{\varepsilon m, R}[p, q]\right)\right\|_{\varepsilon}\left|\alpha_{p, q, R}\right| \varepsilon^{(2 p+q) / 4} . \\
& \leqslant \kappa \varepsilon^{(m+1) / 4} \sum_{0 \leqslant 2 p+q \leqslant 2+3 n}
\end{aligned}
$$

Аналогично, пусть $U_{L}^{\varepsilon}$ обозначает решение задачи $(40)$ с заменой $\widehat{f}$ на

$$
\widehat{f}=\sum_{0 \leqslant 2 p+q \leqslant 2+3 n} \alpha_{p, q, L}(1-x) C_{u}(x)^{2 p} y^{q} .
$$


Тогда находим, что существует постоянная $\kappa>0$, не зависящая от $\varepsilon$, такая, что

$$
\left\|U_{L}^{\varepsilon}-\sum_{0 \leqslant 2 p+q \leqslant 2+3 n} \alpha_{p, q, L} \bar{\xi}_{\varepsilon m, L}[p, q]\right\|_{\varepsilon} \leqslant \kappa \varepsilon^{(m+1) / 4} \sum_{0 \leqslant 2 p+q \leqslant 2+3 n}\left|\alpha_{p, q, L}\right| \varepsilon^{(2 p+q) / 4} .
$$

Из теоремы 3 для решения $V^{\varepsilon}$ задачи (1) с заменой $f$ на $\widetilde{f}=f-\widehat{f}$, где $\widehat{f}$ определено в (167), т. е. $\widetilde{f}$ удовлетворяет условиям совместности (37) с $\mu=2$, замечаем, что

$$
\left\|V^{\varepsilon}-\widetilde{u}_{\varepsilon n}-\widetilde{\theta}_{\varepsilon n}\right\|_{\varepsilon} \leqslant \kappa \varepsilon^{n+1} .
$$

Производя суперпозицию решений, получим $u^{\varepsilon}=U_{R}^{\varepsilon}+U_{L}^{\varepsilon}+V^{\varepsilon}$, где $u^{\varepsilon}-$ то самое решение (1), которое представляет для нас интерес. Полагая $m=4 n+3$ в (173) и (174), получим утверждение теоремы. Теорема 8 доказана.

В зависимости от числа выполненных условий совместности (37), основную теорему можно уточнить, как показывает следующее замечание.

ЗАмЕчАНИЕ 4. Если предположить, что выполняются условия совместности (37), т. е. $\alpha_{p, q, L}=\alpha_{p, q, R}=0$ для $0 \leqslant 2 p+q \leqslant r$ при некотором $r \geqslant 0$, то верхняя граница оценки (173) заменяется на

$$
\kappa \varepsilon^{(m+1) / 4} \sum_{r+1 \leqslant 2 p+q \leqslant 2+3 n}\left|\alpha_{p, q, R}\right| \varepsilon^{(2 p+q) / 4} \leqslant \kappa \varepsilon^{(m+r+2) / 4} .
$$

Тогда для решения $u^{\varepsilon}$ задачи (1) имеем:

$$
\begin{array}{r}
\| u^{\varepsilon}-\widetilde{u}_{\varepsilon n}-\widetilde{\theta}_{\varepsilon n}-\sum_{r+1 \leqslant 2 p+q \leqslant 2+3 n}\left(\alpha_{p, q, L} \bar{\xi}_{\varepsilon m, L}[p, q]\right. \\
\left.+\alpha_{p, q, R} \bar{\xi}_{\varepsilon m, R}[p, q]\right) \|_{\varepsilon} \leqslant \kappa\left(\varepsilon^{n+1}+\varepsilon^{(m+r+2) / 4}\right) .
\end{array}
$$

Конечно, из $(38)$, если $r \geqslant 2+3 n$, можно отбросить член $\kappa \varepsilon^{(m+r+2) / 4}$ из $(175)$.

В частности, для $n=0$ можно произвести анализ сходимости с минимальным количеством асимптотических членов, используя теоремы 1,2 и 4 . Это обсуждается в следующем пункте.

6.2. Дополнения к порядку $n=0$. Напомним и введем следующие определения:

$$
\begin{aligned}
u^{0} & =\int_{y}^{C_{u}(x)} f(x, s) d s, \\
\bar{\theta}^{0} & =-u^{0}(\cos \eta, \sin \eta) \exp \left(\frac{\sin \eta}{\varepsilon} \xi\right) \delta(\xi) \chi_{[\pi, 2 \pi]}(\eta), \\
u_{ \pm}^{* * 0} & =(1-\rho(x) \check{\rho}(x))(1 \pm x)\left(y-C_{u}(x)\right), \\
\theta_{ \pm}^{* * 0} & =-u_{ \pm}^{* * 0}(\cos \eta, \sin \eta) \exp \left(\frac{\sin \eta}{\varepsilon} \xi\right) \delta(\xi) \chi_{[\pi, 2 \pi]}(\eta) .
\end{aligned}
$$

Докажем следующую теорему о сходимости. Здесь заметим, что результат о сходимости (185) много лучше, чем (26). 
Теорема 9. Пусть $u^{\varepsilon}$ - решение задачи (1). Тогда следующая оченка выполняется для произвольной $f$ :

$$
\left\|u^{\varepsilon}-u^{0}-\bar{\theta}^{0}-\bar{\vartheta}^{0}\right\|_{\varepsilon} \leqslant \kappa \varepsilon^{1 / 2}(-\ln \varepsilon)^{1 / 2},
$$

где

$$
\bar{\vartheta}^{0}=2^{-1}\left(f(-1,0) \theta_{-}^{* * 0}+f(1,0) \theta_{+}^{* * 0}\right) \quad c \sigma=\varepsilon^{1 / 3} .
$$

При справедливости предположсения

$$
f=0 \quad \text { в }( \pm 1,0)
$$

выполняется следующая оценка:

$$
\left\|u^{\varepsilon}-u^{0}-\bar{\theta}^{0}\right\|_{\varepsilon} \leqslant \kappa \varepsilon(-\ln \varepsilon)^{1 / 2} .
$$

ДокАзАТЕЛЬСтво. Сначала напомним, что $\widetilde{u}^{0}$ определено в (3) с заменой $f$ на $\tilde{f}$, т. е.

$$
\widetilde{u}^{0}=\int_{y}^{C_{u}(x)} \tilde{f}(x, s) d s
$$

где $\widetilde{f}=f-\widehat{f}$. Здесь $\widehat{f}-$ сумма тейлоровских разложений $f$ до порядка 2 в точках $(1,0)$ и $(-1,0)$, т. е.

$$
\widehat{f}=\sum_{0 \leqslant 2 p+q \leqslant 2}\left(\alpha_{p, q, L}(1-x) C_{u}(x)^{2 p} y^{q}+\alpha_{p, q, R}(1+x) C_{u}(x)^{2 p} y^{q}\right),
$$

и также напомним, что

$$
\widetilde{\theta}^{0}=-\widetilde{u}^{0}(\cos \eta, \sin \eta) \exp ((\sin \eta) \bar{\xi}) \delta(\xi) \chi_{[\pi, 2 \pi]}(\eta) .
$$

Пусть $V^{\varepsilon}$ обозначает решение задачи (1) с заменой $f$ на $\tilde{f}=f-\widehat{f}$. Из теоремы 3 для $n=0$ тогда следует, что

$$
\left\|V^{\varepsilon}-\widetilde{u}^{0}-\widetilde{\theta}^{0}\right\|_{\varepsilon} \leqslant \kappa \varepsilon
$$

Пусть $U_{L}^{\varepsilon}, U_{R}^{\varepsilon}$ обозначают решения задачи (40) с заменой $\widehat{f}$ на

$$
\widehat{f}=\sum_{0 \leqslant 2 p+q \leqslant 2} \alpha_{p, q, L}(1-x) C_{u}(x)^{2 p} y^{q}, \quad \widehat{f}=\sum_{0 \leqslant 2 p+q \leqslant 2} \alpha_{p, q, R}(1+x) C_{u}(x)^{2 p} y^{q}
$$

соответственно. Теорема 4 для четного $q$ позволяет сделать следующее наблюдение. Для $(p, q)=(0,0),(0,2),(1,0)$, т. е. $2 p+q=0,2$, пусть $W_{R}^{\varepsilon}[p, q]-$ решение задачи $(47)$, а $u^{* 0}=u_{R}^{* 0}[p, q]$ задано формулой (50) при $j=0$, и пусть $\bar{\theta}^{* 0}=\bar{\theta}_{R}^{* 0}[p, q]=\theta^{* 0} \delta(\xi)-$ приближенная форма для $\theta^{0}$ из (56), тогда

$$
\begin{aligned}
&\left\|W_{R}^{\varepsilon}[p, q]-u_{R}^{* 0}[p, q]-\bar{\theta}_{R}^{* 0}[p, q]\right\|_{\varepsilon} \\
& \leqslant \kappa \begin{cases}\varepsilon \sigma^{-1}+\min \left\{\varepsilon^{1 / 2}(-\ln \sigma)^{1 / 2}, \varepsilon \sigma^{-2}\right\}, & \text { если } 2 p+q=0, \\
\min \left\{\varepsilon^{1 / 2}, \varepsilon(-\ln \sigma)^{1 / 2}\right\}, & \text { если } 2 p+q=2 .\end{cases}
\end{aligned}
$$


Пусть $W_{L}^{\varepsilon}[p, q]$ - решение задачи $(47)$ с заменой $f^{*}$ на $f^{*}=\rho(x) \check{\rho}(x)(1-x) \times$ $C_{u}(x)^{2 p} y^{q}$. Аналогично определим соответствующую предельную функцию $u_{L}^{* 0}[p, q]$ и нулевой пограничный слой $\bar{\theta}_{L}^{* 0}[p, q]$.

Пусть $S_{R}^{\varepsilon}[p, q]$ - решение задачи $(66)$, и пусть $\bar{\varphi}^{, 0}=\bar{\varphi}_{R}^{, 0}[p, q]$ - соответствующий нулевой параболический пограничный слой. В предположении, что выполняется (158), из теоремы 6 заключаем, что существует постоянная $\kappa>0$, не зависящая от $\sigma, \varepsilon$, такая, что

$$
\left\|S_{R}^{\varepsilon}[p, q]-\bar{\varphi}_{R}^{, 0}[p, q]\right\|_{\varepsilon} \leqslant \kappa \varepsilon^{-1 / 2} \sigma^{2 p+q+3} .
$$

Пусть $S_{L}^{\varepsilon}[p, q]$ - решение задачи $(66)$ с заменой $\widehat{f}-f^{*}$ на $\widehat{f}-f^{*}=(1-$ $\rho(x) \check{\rho}(x))(1-x) C_{u}(x)^{2 p} y^{q}$. Аналогично определим нулевой параболический пограничный слой $\bar{\varphi}_{L}^{, 0}[p, q]$.

Пусть $U_{L}^{\varepsilon}[0,1], U_{R}^{\varepsilon}[0,1]$ обозначают решения задачи (40), где $\widehat{f}$ заменена на

$$
\widehat{f}=(1-x) C_{u}(x)^{2 p} y^{q}=(1-x) y, \quad \widehat{f}=(1+x) C_{u}(x)^{2 p} y^{q}=(1+x) y
$$

соответственно. Из теоремы 4 мы получим следующий результат о сходимости. Для $(p, q)=(0,1)$, т. е. $2 p+q=1$, из (58) следует, что

$$
\left\|U_{l}^{\varepsilon}[0,1]-u_{l}^{0}[0,1]\right\|_{\varepsilon} \leqslant \kappa \varepsilon, \quad l=R, L,
$$

где

$$
\begin{aligned}
& u_{R}^{0}[0,1]=\int_{y}^{C_{u}(x)}(1+x) s d s=\frac{1}{2}(1+x)\left(1-x^{2}-y^{2}\right), \\
& u_{L}^{0}[0,1]=\int_{y}^{C_{u}(x)}(1-x) s d s=\frac{1}{2}(1-x)\left(1-x^{2}-y^{2}\right) .
\end{aligned}
$$

Производя суперпозицию решений, находим

$$
U_{R}^{\varepsilon}=\sum_{\substack{0 \leqslant 2 p+q \leqslant 2 \\ 2 p+q \neq 1}} \alpha_{p, q, R}\left(W_{R}^{\varepsilon}[p, q]+S_{R}^{\varepsilon}[p, q]\right)+\sum_{2 p+q=1} \alpha_{p, q, R} U_{R}^{\varepsilon}[p, q] .
$$

Комбинируя вышеприведенные наблюдения, находим, что

$$
\begin{aligned}
& \left\|U_{R}^{\varepsilon}-\sum_{\substack{0 \leqslant 2 p+q \leqslant 2 \\
2 p+q \neq 1}} \alpha_{p, q, R}\left(u_{R}^{* 0}[p, q]+\bar{\theta}_{R}^{* 0}[p, q]+\bar{\varphi}_{R}^{, 0}[p, q]\right)-\sum_{2 p+q=1} \alpha_{p, q, R} u_{R}^{0}[p, q]\right\|_{\varepsilon} \\
& \leqslant \kappa \sum_{2 p+q=0}\left|\alpha_{p, q, R}\right|\left(\varepsilon \sigma^{-1}+\min \left\{\varepsilon^{1 / 2}(-\ln \sigma)^{1 / 2}, \varepsilon \sigma^{-2}\right\}+\varepsilon^{-1 / 2} \sigma^{3}\right) \\
& \quad+\kappa \sum_{2 p+q=1}\left|\alpha_{p, q, R}\right| \varepsilon+\kappa \sum_{2 p+q=2}\left|\alpha_{p, q, R}\right|\left(\min \left\{\varepsilon^{1 / 2}, \varepsilon(-\ln \sigma)^{1 / 2}\right\}+\varepsilon^{-1 / 2} \sigma^{5}\right) \\
& = \\
& \quad \operatorname{Err}(R) .
\end{aligned}
$$

Такие же оценки мы получим для $U_{L}^{\varepsilon}$ с $u_{L}^{* 0}[p, q], \bar{\theta}_{L}^{* 0}[p, q], \bar{\varphi}_{L}^{, 0}[p, q]$ и $u_{L}^{0}[p, q]$. Здесь мы аналогично определяем $\operatorname{Err}(L)$, заменяя $\alpha_{p, q, R}$ на $\alpha_{p, q, L}$. 
Так как $u^{\varepsilon}=U_{L}^{\varepsilon}+U_{R}^{\varepsilon}+V^{\varepsilon}$, то комбинируя (189) и (198) и записывая $\bar{\xi}_{l}^{0}[p, q]=$ $u_{l}^{* 0}[p, q]+\bar{\theta}_{l}^{* 0}[p, q]+\bar{\varphi}_{l}^{, 0}[p, q]$ для $2 p+q \neq 1$ и $\bar{\xi}_{l}^{0}[p, q]=u_{l}^{0}[p, q]=u_{l}^{0}[0,1]$ для $2 p+q=1$, где $l=R, L$, находим, что

$$
\begin{aligned}
& \left\|u^{\varepsilon}-\widetilde{u}^{0}-\widetilde{\theta}^{0}-\sum_{0 \leqslant 2 p+q \leqslant 2}\left(\alpha_{p, q, L} \bar{\xi}_{L}^{0}[p, q]+\alpha_{p, q, R} \bar{\xi}_{R}^{0}[p, q]\right)\right\|_{\varepsilon} \\
& \leqslant \kappa(\operatorname{Err}(R)+\operatorname{Err}(L)) .
\end{aligned}
$$

Заметим, что

$$
\widetilde{u}^{0}=\int_{y}^{C_{u}(x)}(f-\widehat{f})(x, s) d s=u^{0}-\sum_{0 \leqslant 2 p+q \leqslant 2}\left(\alpha_{p, q, L} \widehat{u}_{L}^{0}[p, q]+\alpha_{p, q, R} \widehat{u}_{R}^{0}[p, q]\right),
$$

где

$$
\widehat{u}_{L}^{0}[p, q]=\int_{y}^{C_{u}(x)}(1-x) C_{u}(x)^{2 p} s^{q} d s, \quad \widehat{u}_{R}^{0}[p, q]=\int_{y}^{C_{u}(x)}(1+x) C_{u}(x)^{2 p} s^{q} d s,
$$

и

$$
\begin{aligned}
\widetilde{\theta}^{0} & =-\widetilde{u}^{0}(\cos \eta, \sin \eta) \exp \left(\frac{\sin \eta}{\varepsilon} \xi\right) \delta(\xi) \chi_{[\pi, 2 \pi]}(\eta) \\
& =\bar{\theta}^{0}-\sum_{0 \leqslant 2 p+q \leqslant 2}\left(\alpha_{p, q, L} \widehat{\theta}_{L}^{0}[p, q]+\alpha_{p, q, R} \widehat{\theta}_{R}^{0}[p, q]\right)
\end{aligned}
$$

где для $l=L, R$

$$
\widehat{\theta}_{l}^{0}[p, q]=-\widehat{u}_{l}^{0}[p, q](\cos \eta, \sin \eta) \exp \left(\frac{\sin \eta}{\varepsilon} \xi\right) \delta(\xi) \chi_{[\pi, 2 \pi]}(\eta) .
$$

Из (195) и (196) легко видеть, что $\widehat{u}_{l}[0,1]=u_{l}^{0}[0,1]$ и $\widehat{u}_{l}[0,1](\cos \eta, \sin \eta)=$ $u_{l}^{0}(\cos \eta, \sin \eta)=0, l=R, L$. Тогда из (199) находим, что

$$
\begin{gathered}
\left\|u^{\varepsilon}-u^{0}-\bar{\theta}^{0}-\sum_{\substack{0 \leqslant 2 p+q \leqslant 2 \\
2 p+q \neq 1}}\left(\alpha_{p, q, L} \xi_{L}^{* * 0}[p, q]+\alpha_{p, q, R} \xi_{R}^{* * 0}[p, q]\right)\right\|_{\varepsilon} \\
\leqslant \kappa(\operatorname{Err}(R)+\operatorname{Err}(L)),
\end{gathered}
$$

где для $l=R, L$

$$
\begin{aligned}
\xi_{l}^{* * 0}[p, q] & =-\widehat{u}_{l}[p, q]-\widehat{\theta}_{l}[p, q]+\bar{\xi}_{l}^{0}[p, q] \\
& =-\widehat{u}_{l}[p, q]-\widehat{\theta}_{l}[p, q]+u_{l}^{* 0}[p, q]+\bar{\theta}_{l}^{* 0}[p, q]+\bar{\varphi}_{l}^{, 0}[p, q] .
\end{aligned}
$$

Заметим затем, что

$$
\xi_{R}^{* * 0}[p, q]=-u_{R}^{* * 0}[p, q]-\theta_{R}^{* * 0}[p, q]+\bar{\varphi}_{R}^{, 0}[p, q],
$$

где

$$
\begin{aligned}
& u_{R}^{* * 0}[p, q]=-\int_{y}^{C_{u}(x)}(1-\rho(x) \check{\rho}(x))(1+x) C_{u}(x)^{2 p} s^{q} d s \\
& \theta_{R}^{* * 0}[p, q]=-u_{R}^{* * 0}[p, q](\cos \eta, \sin \eta) \exp \left(\frac{\sin \eta}{\varepsilon} \xi\right) \delta(\xi) \chi_{[\pi, 2 \pi]}(\eta)
\end{aligned}
$$


Сначала заметим, что выполнены поточечные оценки

$$
\begin{aligned}
\left|\partial_{y}^{m} u_{R}^{* * 0}[p, q]\right| \leqslant & \kappa(1-\rho(x) \check{\rho}(x)) C_{u}(x)^{2 p+q+1-m}, \quad m=0,1, \\
\left|\partial_{x} u_{R}^{* * 0}[p, q]\right| \leqslant & \kappa(1-\rho(x) \check{\rho}(x)) C_{u}(x)^{2 p+q-1} \\
& +\kappa(1-\rho(x) \check{\rho}(x))_{x} C_{u}(x)^{2 p+q+1} .
\end{aligned}
$$

Используя (45) и (43), находим, что

$$
\begin{aligned}
\left|u_{R}^{* * 0}[p, q]\right| & \leqslant \kappa \tilde{\sigma}^{(2 p+q+1) / 2} \chi_{[-1,-1+\widetilde{\sigma}] \cup[1-\widetilde{\sigma}, 1]}(x), \\
\left|\nabla u_{R}^{* * 0}[p, q]\right| & \leqslant \kappa\left(\widetilde{\sigma}^{(2 p+q-1) / 2}+C_{u}(x)^{2 p+q-1}\right) \chi_{[-1,-1+\widetilde{\sigma}] \cup[1-\widetilde{\sigma}, 1]}(x) .
\end{aligned}
$$

Пусть $D_{\sigma}=D \cap(\{-1<x<-1+\widetilde{\sigma}\} \cup\{1-\widetilde{\sigma}<x<1\})$. Сначала заключаем из (46), что

$$
\begin{aligned}
\left|C_{u}(x)^{2 p+q-1}\right|_{L^{2}\left(D_{\sigma}\right)} & \leqslant \kappa\left(\int_{1-\widetilde{\sigma}}^{1} C_{u}(x)^{2(2 p+q-1)+1} d x\right)^{1 / 2} \\
& \leqslant \kappa \sigma^{2 p+q+1 / 2}
\end{aligned}
$$

Используя то обстоятельство, что $|1|_{L^{2}\left(D_{\sigma}\right)} \leqslant \kappa \sigma^{3 / 2}$, заключаем из (18), что

$$
\begin{gathered}
\left|u_{R}^{* * 0}[p, q]\right|_{L^{2}} \leqslant \kappa \sigma^{2 p+q+1}|1|_{L^{2}\left(D_{\sigma}\right)} \leqslant \kappa \sigma^{2 p+q+5 / 2} \\
\left|u_{R}^{* * 0}[p, q]\right|_{H^{1}} \leqslant \kappa\left(\sigma^{2 p+q-1}|1|_{L^{2}\left(D_{\sigma}\right)}+\left|C_{u}(x)^{2 p+q-1}\right|_{L^{2}\left(D_{\sigma}\right)}\right) \\
\leqslant \kappa \sigma^{2 p+q+1 / 2}
\end{gathered}
$$

и, таким образом,

$$
\left\|u_{R}^{* * 0}[p, q]\right\|_{\varepsilon} \leqslant \kappa\left(\varepsilon^{1 / 2}+\sigma^{2}\right) \sigma^{2 p+q+1 / 2} .
$$

Кроме того, из (209) и (210) мы заключаем, что

$$
\begin{aligned}
& \left|u_{R}^{* * 0}[p, q](\cos \eta, \sin \eta)\right| \leqslant \kappa|\sin \eta|^{2 p+q+1} \chi_{[-\sigma, \sigma] \cup[\pi-\sigma, \pi+\sigma]}(\eta), \\
& \left|\partial_{\eta} u_{R}^{* * 0}[p, q](\cos \eta, \sin \eta)\right| \\
& \quad \leqslant \kappa\left|\partial_{x} u_{R}^{* * 0}[p, q](\cos \eta, \sin \eta)\right||\sin \eta|+\kappa\left|\partial_{y} u_{R}^{* * 0}[p, q](\cos \eta, \sin \eta)\right| \\
& \quad \leqslant \kappa\left(|\sin \eta|^{2 p+q-1}+\widetilde{\sigma}^{-1}|\sin \eta|^{2 p+q+1}\right) \chi_{[-\sigma, \sigma] \cup[\pi-\sigma, \pi+\sigma]}(\eta) .
\end{aligned}
$$

Отсюда получим оценки для (208):

$$
\begin{aligned}
\left|\theta_{R}^{* * 0}[p, q]\right|_{L^{2}} & \leqslant \kappa \varepsilon^{1 / 2} \sigma^{2 p+q+1}, \\
\left|\partial_{\eta} \theta_{R}^{* * 0}[p, q]\right|_{L^{2}} & \leqslant \kappa \varepsilon^{1 / 2} \sigma^{2 p+q-1}, \quad \text { если } 2 p+q>1, \\
\left|\partial_{\xi} \theta_{R}^{* * 0}[p, q]\right|_{L^{2}} & \leqslant \kappa \varepsilon^{-1 / 2} \sigma^{2 p+q+2},
\end{aligned}
$$

и, таким образом, используя (158), имеем

$$
\left\|\theta_{R}^{* * 0}[p, q]\right\|_{\varepsilon} \leqslant \kappa \sigma^{2 p+q+2}, \quad \text { если } 2 p+q>1 .
$$

Из (150) следует, что для $p, q \geqslant 0$

$$
\left\|\bar{\varphi}_{R}^{, 0}[p, q]\right\|_{\varepsilon} \leqslant \kappa \sigma^{2 p+q+2} .
$$


Из (204), (216), (222) и (223) заключаем, что

$$
\begin{aligned}
& \left\|u^{\varepsilon}-u^{0}-\bar{\theta}^{0}-\left(\alpha_{0,0, L} \theta_{L}^{* * 0}[0,0]+\alpha_{0,0, R} \theta_{R}^{* * 0}[0,0]\right)\right\|_{\varepsilon} \\
& \leqslant \kappa(\operatorname{Err}(R)+\operatorname{Err}(L))+\kappa \sum_{2 p+q=0}\left(\left|\alpha_{0,0, R}\right|+\left|\alpha_{0,0, L}\right|\right)\left(\varepsilon^{1 / 2} \sigma^{1 / 2}+\sigma^{2}\right) \\
& \quad+\kappa \sum_{2 p+q=2}\left(\left|\alpha_{p, q, R}\right|+\left|\alpha_{p, q, L}\right|\right)\left(\varepsilon^{1 / 2} \sigma^{1 / 2}+\sigma^{2}\right) \sigma^{2} \\
& \quad=: \operatorname{Err}^{\prime} .
\end{aligned}
$$

Полагая $\sigma=\varepsilon^{1 / 3}$, находим, что $\operatorname{Err}^{\prime} \leqslant \kappa \varepsilon^{1 / 2}(-\ln \varepsilon)^{1 / 2}$, и это влечет $(182)$.

Предположив, что

$$
f=0 \text { в }( \pm 1,0), \quad \text { т. е. } \alpha_{0,0, R}=\alpha_{0,0, L}=0,
$$

и полагая $\sigma=\varepsilon^{1 / 3}$, находим, что $\operatorname{Err}^{\prime} \leqslant \kappa \varepsilon(-\ln \varepsilon)$ и, следовательно, оценка $(224)$ влечет (185). Теорема 9 доказана.

ЗАмЕЧАНИЕ 5. Если предположить, что

$$
\begin{gathered}
\frac{\partial^{p+q} f}{\partial x^{p} \partial y^{q}}=0 \quad \text { в }( \pm 1,0) \text { для } 2 p+q=0,2, p, q \geqslant 0, \\
\text { т. е. } f=\frac{\partial^{2} f}{\partial y^{2}}=\frac{\partial f}{\partial x}=0 \quad \text { в }( \pm 1,0),
\end{gathered}
$$

то, так как $\operatorname{Err}^{\prime} \leqslant \kappa \varepsilon$, оценка $(224)$ влечет

$$
\left\|u^{\varepsilon}-u^{0}-\bar{\theta}^{0}\right\|_{\varepsilon} \leqslant \kappa \varepsilon .
$$

Складывая параболические пограничные слои $\bar{\varphi}_{-}^{, 0}, \bar{\varphi}_{+}^{, 0}$ как в $(229)$, можно улучшить результат о сходимости (182).

Теорема 10. Пусть и $^{\varepsilon}-$ решение задачи (1). Тогда имеют место следующие оченки для произвольной $f$ :

$$
\left\|u^{\varepsilon}-u^{0}-\bar{\theta}^{0}-\bar{\vartheta}^{0}\right\|_{\varepsilon} \leqslant \kappa \varepsilon^{4 / 7}
$$

¿əe

$$
\bar{\vartheta}^{0}=2^{-1}\left(f(-1,0)\left(\theta_{-}^{* * 0}+\bar{\varphi}_{-}^{, 0}\right)+f(1,0)\left(\theta_{+}^{* * 0}+\bar{\varphi}_{+}^{, 0}\right)\right) \quad c \sigma=\varepsilon^{3 / 14} .
$$

Здесъ $\bar{\varphi}_{+}^{, 0}=\bar{\varphi}_{R}^{, 0}[0,0]$ и $\bar{\varphi}_{-}^{, 0}=\bar{\varphi}_{L}^{, 0}[0,0]-$ нулевые параболические пограничные слои, отвечающие (66) с заменой $\widehat{f}-f^{*}$ соответственно на $(1-\rho(x) \check{\rho}(x))(1+x)$ $u(1-\rho(x) \check{\rho}(x))(1-x)$.

ЗАмечание 6 . Заметим, что (228) влечет за собой $H^{1}$-сходимость, т. е.

$$
\left\|u^{\varepsilon}-u^{0}-\bar{\theta}^{0}-\bar{\vartheta}^{0}\right\|_{H^{1}(D)} \leqslant \kappa \varepsilon^{1 / 14} .
$$

ДокАЗАТЕЛЬСтво теоремы 10 . Заменяя $\bar{\varphi}_{R}^{, 0}[p, q]$ в доказательстве теоремы 9 на $\bar{\varphi}_{\varepsilon m R}[p, q]$, мы улучшаем результат о сходимости (182). Из теоремы 6 для $n=m$ следует справедливость следующей оценки:

$$
\left\|S_{R}^{\varepsilon}[p, q]-\bar{\varphi}_{\varepsilon m R}[p, q]\right\|_{\varepsilon} \leqslant \kappa\left(\varepsilon^{-1} \sigma^{6}\right)^{m / 2} \varepsilon^{-1 / 2} \sigma^{2 p+q+3},
$$


где $\bar{\varphi}_{\varepsilon m R}[p, q]=\sum_{i=0}^{m} \sigma^{2 i} \bar{\varphi}_{R}^{2 i}[p, q]$ и $\bar{\varphi}_{R}^{2 i}[p, q]=\bar{\varphi}^{2 i}[p, q]$ - параболические пограничные слои из (C1), (C2) в разделе 5 , соответствующие задаче (66). Тогда для $\operatorname{Err}(R)$ в (198) справедливы следующие оценки:

$$
\begin{aligned}
& \operatorname{Err}(R) \leqslant \kappa \sum_{2 p+q=0}\left|\alpha_{p, q, R}\right|\left(\varepsilon \sigma^{-1}+\min \left\{\varepsilon^{1 / 2}(-\ln \sigma)^{1 / 2}, \varepsilon \sigma^{-2}\right\}\right. \\
& \left.\quad+\left(\varepsilon^{-1} \sigma^{6}\right)^{m / 2} \varepsilon^{-1 / 2} \sigma^{3}\right)+\kappa \sum_{2 p+q=1}\left|\alpha_{p, q, R}\right| \varepsilon \\
& \quad+\kappa \sum_{2 p+q=2}\left|\alpha_{p, q, R}\right|\left(\min \left\{\varepsilon^{1 / 2}, \varepsilon(-\ln \sigma)^{1 / 2}\right\}+\left(\varepsilon^{-1} \sigma^{6}\right)^{m / 2} \varepsilon^{-1 / 2} \sigma^{5}\right) .
\end{aligned}
$$

Как и в (224), находим, что

$$
\begin{aligned}
& \left\|u^{\varepsilon}-u^{0}-\bar{\theta}^{0}-\left(\alpha_{0,0, L}\left(\theta_{L}^{* * 0}+\bar{\varphi}_{\varepsilon m L}\right)[0,0]+\alpha_{0,0, R}\left(\theta_{R}^{* * 0}+\bar{\varphi}_{\varepsilon m R}\right)[0,0]\right)\right\|_{\varepsilon} \\
& \leqslant \kappa(\operatorname{Err}(R)+\operatorname{Err}(L))+\kappa \sum_{2 p+q=0}\left(\left|\alpha_{0,0, R}\right|+\left|\alpha_{0,0, L}\right|\right) \varepsilon^{1 / 2} \sigma^{1 / 2} \\
& \quad+\kappa \sum_{2 p+q=2}\left(\left|\alpha_{p, q, R}\right|+\left|\alpha_{p, q, L}\right|\right)\left(\varepsilon^{1 / 2} \sigma^{1 / 2}+\sigma^{3 / 2}\right) \sigma^{2} \\
& \quad: \operatorname{Err}^{\prime} .
\end{aligned}
$$

Полагая $\varepsilon \sigma^{-2}=\left(\varepsilon^{-1} \sigma^{6}\right)^{m / 2} \varepsilon^{-1 / 2} \sigma^{3}$ и, следовательно,

$$
\sigma=\varepsilon^{(m+3) /(2(3 m+5))} \quad(\text { это } \sigma \text { согласуется с }(158)),
$$

находим, что

$$
\operatorname{Err}^{\prime} \leqslant \kappa \varepsilon^{(2 m+2) /(3 m+5)}+\kappa \varepsilon^{(7 m+13) /(4(3 m+5))}, \quad m \geqslant 1 .
$$

Из (150) следует, что для $p, q \geqslant 0$

$$
\left\|\bar{\varphi}_{R}^{, 2 i}[0,0]\right\|_{\varepsilon} \leqslant \kappa \varepsilon^{-i / 2} \sigma^{2+i},
$$

и, значит,

$$
\left\|\sum_{i=1}^{m} \sigma^{2 i} \bar{\varphi}_{R}^{, 2 i}[0,0]\right\|_{\varepsilon} \leqslant \kappa \sum_{i=1}^{m} \varepsilon^{-i / 2} \sigma^{2+3 i} \stackrel{(158)}{\leqslant} \kappa \varepsilon^{-1 / 2} \sigma^{5} .
$$

Затем заключаем из (233), что

$$
\left\|u^{\varepsilon}-u^{0}-\bar{\theta}^{0}-\bar{\vartheta}^{0}\right\|_{\varepsilon} \leqslant \kappa \varepsilon^{(2 m+2) /(3 m+5)}+\kappa \varepsilon^{(7 m+13) /(4(3 m+5))}+\kappa \varepsilon^{(m+5) /(3 m+5)},
$$

где $\bar{\vartheta}^{0}$ на этот раз определяется формулой

$$
\begin{aligned}
\bar{\vartheta}^{0}= & 2^{-1}\left(f(-1,0)\left(\theta_{L}^{* * 0}+\bar{\varphi}_{L}^{, 0}\right)[0,0]\right. \\
& \left.+f(1,0)\left(\theta_{R}^{* * 0}+\bar{\varphi}_{R}^{, 0}\right)[0,0]\right) \quad \text { c } \sigma=\varepsilon^{(m+3) /(2(3 m+5))}, \quad m \geqslant 1 .
\end{aligned}
$$

Полагая $m=3$ (откуда следует, что $\varepsilon^{(2 m+2) /(3 m+5)}=\varepsilon^{(m+5) /(3 m+5)}=\varepsilon^{4 / 7}>$ $\left.\varepsilon^{(7 m+13) /(4(3 m+5))}\right)$, завершаем доказательство теоремы. 


\section{7. Заключительные замечания}

Для того чтобы построить пограничные слои, произведенные уравнением (1) с произвольной гладкой функцией $f$, мы рассмотрели разложение $f=f-\widehat{f}+$ $\rho(x) \check{\rho}(x) \widehat{f}+(1-\rho(x) \check{\rho}(x)) \hat{f}$. Анализ для $f$, замененной на $f-\widehat{f}$ или $\rho(x) \check{\rho}(x) \widehat{f}$, проделан в разделах 2 и 3 и заимствован из [24] и [25]. Затем мы изучили случай, когда $f=(1-\rho(x) \check{\rho}(x)) \widehat{f}$ и возникают так называемые параболические пограничные слои. Отсюда следуют результаты для $f$ общего вида (см. раздел 6). Мы доказали результаты о сходимости для любого порядка.

Параболические пограничные слои в характеристических точках $( \pm 1,0)$ полностью изучены, и для них получены оценки в разделах 4 и 5 . Эти параболические пограничные слои не выражаются в явном виде, однако в (95) для них получены энергетические оценки при помощи керн-функций $M_{l, d}$. Мы надеемся, что техника изучения параболических слоев, не имеющих явного выражения, развитая в этой статье, будет полезна для изучения других параболических слоев, явное выражение для которых нельзя получить. В [13] приводится выражение для промежуточных пограничных слоев (68) порядка 0, 1 через функции Эйри, но, к сожалению, этот замечательный результат не удается воспроизвести для более высоких порядков, поскольку в нашем распоряжении нет явных выражений для корректоров пограничных слоев в высших порядках. Наш подход позволяет привести оценки для любого пограничного слоя, определяемого уравнением пограничного слоя.

\section{Список литературы}

[1] I. Andronov, D. Bouche, F. Molinet, Asymptotic and hybrid methods in electromagnetics, IEE Electromagnet. Waves Ser., 48, Institution of Electrical Engineers (IEE), London, 2005, xii+249 pp.

[2] C. Bardos, "Problèmes aux limites pour les équations aux dérivées partielles du premier ordre à coefficients réels; théorèmes d'approximation; application à l'équation de transport", Ann. Sci. École Norm. Sup. (4), 3:2 (1970), 185-233.

[3] G.K. Batchelor, An introduction to fluid dynamics, 2nd paperback ed., Cambridge Math. Lib., Cambridge Univ. Press, Cambridge, 1999, xviii+615 pp.

[4] D. Bouche, O. Lafitte, "Comparaison couche limite - analyse microlocale", Asymptot. Anal. (to appear).

[5] D. Bouche, F. Molinet, Méthodes asymptotiques en électromagnétisme, with a preface by R. Dautray, Math. Appl. (Berlin), 16, Springer-Verlag, Paris, 1994, xviii+416 pp.

[6] K. W. Chang, F. A. Howes, Nonlinear singular perturbation phenomena: theory and applications, Appl. Math. Sci., 56, Springer-Verlag, New York, 1984, viii+180 pp.

[7] M. G. Crandall, P.-L. Lions, "Viscosity solutions of Hamilton-Jacobi equations", Trans. Amer. Math. Soc., 277:1 (1983), 1-42.

[8] P. G. Drazin, W.H. Reid, Hydrodynamic stability, with a foreword by J. Miles, 2nd ed., Cambridge Math. Lib., Cambridge Univ. Press, Cambridge, 2004, xx+605 pp.

[9] W. Eckhaus, "Boundary layers in linear elliptic singular perturbation problems", SIAM Rev., 14:2 (1972), 225-270.

[10] W. Eckhaus, Asymptotic analysis of singular perturbations, Stud. Math. Appl., 9, North-Holland Publishing Co., Amsterdam-New York, 1979, xi+287 pp.

[11] W. Eckhaus, E. M. de Jager, "Asymptotic solutions of singular perturbation problems for linear differential equations of elliptic type", Arch. Ration. Mech. Anal., 23:1 (1966), 26-86. 
[12] G.-M. Gie, M. Hamouda, R. Temam, "Asymptotic analysis of the Stokes problem on general bounded domains: the case of a characteristic boundary", Appl. Anal., 89:1 (2010), 49-66.

[13] J. Grasman, On the birth of boundary layers, Math. Centre Tracts, 36, Mathematisch Centrum, Amsterdam, 1971, iv+137 pp.

[14] M. Hamouda, C.-Y. Jung, R. Temam, "Boundary layers for the 2D linearized primitive equations", Commun. Pure Appl. Anal., 8:1 (2009), 335-359.

[15] M. Hamouda, C.-Y. Jung, R. Temam, "Asymptotic analysis for the 3D primitive equations in a channel", Discrete Contin. Dyn. Syst. Ser. S, 6:2 (2013), 401-422.

[16] M. Hamouda, R. Temam, "Some singular perturbation problems related to the Navier-Stokes equations", Advances in deterministic and stochastic analysis, World Sci. Publ., Hackensack, NJ, 2007, 197-227.

[17] M. Hamouda, R. Temam, "Boundary layers for the Navier-Stokes equations. The case of a characteristic boundary", Georgian Math. J., 15:3 (2008), 517-530.

[18] C.-Y. Jung, T.B. Nguyen, "Semi-analytical numerical methods for convectiondominated problems with turning points", Int. J. Numer. Anal. Model., 10:2 (2013), 314-332.

[19] C.-Y. Jung, M. Petcu, R. Temam, "Singular perturbation analysis on a homogeneous ocean circulation model", Anal. Appl. (Singap.), 9:3 (2011), 275-313.

[20] C.-Y. Jung, R. Temam, "Numerical approximation of two-dimensional convection-diffusion equations with multiple boundary layers", Int. J. Numer. Anal. Model., 2:4 (2005), 367-408.

[21] C.-Y. Jung, R. Temam, "On parabolic boundary layers for convection-diffusion equations in a channel: analysis and numerical applications", J. Sci. Comput., 28:2-3 (2006), 361-410.

[22] C.-Y. Jung, R. Temam, "Asymptotic analysis for singularly perturbed convectiondiffusion equations with a turning point", J. Math. Phys., 48:6 (2007), 065301, 27 pp.

[23] C.-Y. Jung, R. Temam, "Interaction of boundary layers and corner singularities", Discrete Contin. Dyn. Syst., 23:1-2 (2009), 315-339.

[24] C.-Y. Jung, R. Temam, "Convection-diffusion equations in a circle: the compatible case", J. Math. Pures Appl. (9), 96:1 (2011), 88-107.

[25] C.-Y. Jung, R. Temam, "Singular perturbations and boundary layer theory for convection-diffusion equations in a circle: the generic noncompatible case", SIAM J. Math. Anal., 44:6 (2012), 4274-4296.

[26] T. Kato, "Remarks on the Euler and Navier-Stokes equations in $\mathbf{R}^{2}$ ", Nonlinear functional analysis and its applications, part 2 (Berkeley, CA, 1983), Proc. Sympos. Pure Math., 45 part 2, Amer. Math. Soc., Providence, RI, 1986, 1-7.

[27] J.B. Keller, S.I. Rubinow, "Asymptotic solutions of eigenvalue problems", Ann. Physics, 9:1 (1960), 24-75.

[28] N. Levinson, "The first boundary value problem for $\varepsilon \Delta u+A(x, y) u_{x}+B(x, y) u_{y}+$ $C(x, y) u=D(x, y)$ for small $\varepsilon "$, Ann. of Math. (2), 51 (1950), 428-445.

[29] J.-L. Lions, Perturbations singulières dans les problèmes aux limites et en contrôle optimal, Lecture Notes in Math., 323, Springer-Verlag, Berlin-New York, 1973, xii+645 pp.

[30] P.-L. Lions, "On the Hamilton-Jacobi-Bellman equations", Acta Appl. Math., 1:1 (1983), 17-41.

[31] M. C. Lombardo, M. Sammartino, "Zero viscosity limit of the Oseen equations in a channel", SIAM J. Math. Anal., 33:2 (2001), 390-410 (electronic).

[32] О.А. Олейник, "О линейных уравнениях второго порядка с неотрицательной характеристической формой", Матем. сб., 69(111):1 (1966), 111-140.

[33] R. E. O'Malley, Jr., "On boundary value problems for a singularly perturbed differential equation with a turning point", SIAM J. Math. Anal., 1:4 (1970), 479-490. 
[34] R. E. O'Malley, Jr., "Singularly perturbed linear two-point boundary value problems", SIAM Rev., 50:3 (2008), 459-482.

[35] L. Prandtl, "Über Flüssigkeitsbewegung bei sehr kleiner Reibung", Verhandlungen des 3. Internationalen Mathematischen Kongress (Heidelberg, 1904), B. G. Teubner, Leipzig, 1905, 484-491.

[36] L. Prandtl, Gesammelte Abhandlungen zur angewandten Mechanik, Hydro- und Aerodynamik, v.1-3, ed. F.W. Riegels, Springer-Verlag, Berlin-Göttingen-Heidelberg, 1961, xx+xiv+xiv+1620 pp.

[37] J.-P. Raymond, "Stokes and Navier-Stokes equations with nonhomogeneous boundary conditions", Ann. Inst. H. Poincaré Anal. Non Linéaire, 24:6 (2007), 921-951.

[38] H.-G. Roos, M. Stynes, L. Tobiska, Numerical methods for singularly perturbed differential equations. Convection-diffusion and flow problems, Springer Ser. Comput. Math., 24, Springer-Verlag, Berlin, 1996, xvi+348 pp.

[39] S.-D. Shih, R. B. Kellogg, "Asymptotic analysis of a singular perturbation problem", SIAM J. Math. Anal., 18:5 (1987), 1467-1511.

[40] M. Stynes, "Steady-state convection-diffusion problems", Acta Numer., 14 (2005), 445-508.

[41] R. Temam, Navier-Stokes equations. Theory and numerical analysis, reprint of the 1984 edition, AMS Chelsea Publishing, Providence, RI, 2001, xiv+408 pp.

[42] R. Temam, X. Wang, "Boundary layers for Oseen's type equation in space dimension three", Russian J. Math. Phys., 5:2 (1997), 227-246.

[43] R. Temam, X. Wang, "Boundary layers associated with incompressible Navier-Stokes equations: the noncharacteristic boundary case", J. Differential Equations, 179:2 (2002), 647-686.

[44] N. M. Temme, "Analytical methods for an elliptic singular perturbation problem in a circle", J. Comput. Appl. Math., 207:2 (2007), 301-322.

[45] F. Verhulst, Methods and applications of singular perturbations. Boundary layers and multiple timescale dynamics, Texts Appl. Math., 50, Springer, New York, 2005, $\mathrm{xvi}+324 \mathrm{pp}$.

[46] М.И. Вишик, Л.А. Люстерник, "Регулярное вырождение и пограничный слой для линейных дифференциальных уравнений с малым параметром", УМН, 12:5(77) (1957), 3-122.

[47] T. von Kármán, "Progress in the statistical theory of turbulence", J. Marine Research, 7 (1948), 252-264; Proc. Nat. Acad. Sci. U. S. A., 34 (1948), 530-539.

[48] W. Wasow, Asymptotic expansions for ordinary differential equations, Pure Appl. Math., 14, Interscience Publishers John Wiley \& Sons, Inc., New York-LondonSydney, 1965, ix +362 pp.

[49] W. Wasow, Linear turning point theory, Appl. Math. Sci., 54, Springer-Verlag, New York, 1985, ix+246 pp.

\section{Чанг-Юоль Юнг}

(Chang-Yeol Jung)

School of Natural Science, Ulsan National Institute of

Science and Technology, Ulsan, Republic of Korea

E-mail: cjung@unist.ac.kr

\section{Роже Темам}

\section{(Roger Temam)}

The Institute for Scientific Computing and Applied

Mathematics, Indiana University, Bloomington, U.S.A.

E-mail: temam@indiana.edu
Поступила в редакцию 25.10 .2013 